\title{
Chaos and correlated avalanches in excitatory neural networks with synaptic plasticity
}

\author{
Fabrizio Pittorino, ${ }^{1,2}$ Miguel Ibáñez-Berganza, ${ }^{2}$ Matteo di Volo, ${ }^{3}$ Alessandro Vezzani ${ }^{4,1}$ and Raffaella Burioni ${ }^{1,2}$ \\ ${ }^{1}$ Dipartimento di Scienze Matematiche, Fisiche e Informatiche, \\ Università di Parma, via G.P. Usberti, 7/A - 43124, Parma, Italy \\ ${ }^{2}$ INFN, Gruppo Collegato di Parma, via G.P. Usberti, 7/A - 43124, Parma, Italy \\ ${ }^{3}$ Group for Neural Theory, Laboratoire de Neurosciences Cognitives, \\ INSERM U960, École Normale Supérieure, Paris, France \\ ${ }^{4}$ IMEM-CNR, Parco Area delle Scienze, 37/A-43124 Parma, Italy
}

\begin{abstract}
A collective chaotic phase with power law scaling of activity events is observed in a disordered mean field network of purely excitatory leaky integrate-and-fire neurons with short-term synaptic plasticity. The dynamical phase diagram exhibits two transitions from quasi-synchronous and asynchronous regimes to the nontrivial, collective, bursty regime with avalanches. In the homogeneous case without disorder, the system synchronizes and the bursty behavior is reflected into a period doubling transition to chaos for a two dimensional discrete map. Numerical simulations show that the bursty chaotic phase with avalanches exhibits a spontaneous emergence of persistent time correlations and enhanced Kolmogorov complexity. Our analysis reveals a mechanism for the generation of irregular avalanches that emerges from the combination of disorder and deterministic underlying chaotic dynamics.
\end{abstract}

Networks of spiking neurons feature a wide range of dynamical collective behaviors, that are believed to be crucial for brain functioning [1]. Next to uncorrelated and asynchronous dynamics, quasi-synchronous phases and regimes of irregular activity have been observed, showing a still unexplained degree of correlation that could encode part of the neural function [2-7]. Understanding the mechanisms that generate such experimentally observed collective behaviors and the transition between them is a major goal in theoretical neuroscience [1, 8-15]. A particularly interesting dynamical signature of collective irregular regimes are avalanches or bursts of spiking neurons with heavy-tailed distributions of activity $[6,16,17]$. Interestingly, in cortical networks, irregular activity at the collective level $[18,19]$ and avalanches characterized by power law distributions have been widely observed both in vitro and in vivo [20-23]. These regimes are thought to be closely related to information processing in the cortex [24-26] and to adaptive [27] and healthy [28] behavior.

Several mechanisms leading to irregular dynamics and bursts in networks of spiking neurons have been proposed. Irregular dynamical phases have been related to a balance between excitatory and inhibitory inputs $[29,30]$ or to a disorder in the network or in the couplings $[11,31]$ as crucial ingredients. Power law distributed avalanches have been attributed to synaptic plasticity with a stochastic noise in the charging [32-36] or to dynamical mechanisms inspired by self organized criticality (SOC) $[27,37,38]$. The balance between excitation and inhibition plays an important role in the latter dynamical regime as well [39], and a relation between uncorrelated dynamics in a network of stochastic units and power law scaling has been proposed [40, 41].

In this Letter we show that correlated irregular dynamics can be observed in homogeneous deterministic networks of $N$ identical purely excitatory spiking neurons endowed with synaptic plasticity, coupled by an all to all, mean field (MF), interaction. In this case, all neurons are synchronized but, for small enough synaptic decay time, the system displays a period doubling transition from a periodic phase to synchronous chaos [42, 43]. Such a transition is determined by the competition among the system time scales in the strong and weak coupling limits. For vanishing synaptic decay time, the dynamics can be reduced to a one dimensional map.

In the presence of disorder in the couplings, we show that the dynamics exhibits three phases, depending on the interaction strength and synaptic decay time. In particular, next to the quasi-synchronous and the asynchronous regimes [44], a phase characterized by power law distributed avalanches emerges in correspondence to the chaotic phase of the homogeneous MF model. Chaos is preserved in this dynamical phase, as confirmed by the computation of the Lyapunov exponents, and it is characterized by the onset of strong temporal correlations and high complexity. Our analysis uncovers a connection between dynamical stability and emergent avalanche activity in the presence of short-term synaptic plasticity, that may go beyond our particular case of study.

We consider a disordered random network of leaky integrate-and-fire (LIF) neurons [45] connected via the Tsodyks-Uziel-Markram (TUM) model for short term synaptic plasticity [46]. Within a Degree based Mean Field approximation (DMF), for each neuron $i=1 \ldots N$ the dynamics is defined by three differential equations:

$$
\begin{aligned}
& \dot{v}_{i}(t)=a-v_{i}(t)+g k_{i} Y(t) \\
& \dot{y}_{i}(t)=-\frac{y_{i}(t)}{\tau_{i n}}+u\left(1-y_{i}(t)-z_{i}(t)\right) S_{i}(t)
\end{aligned}
$$




$$
\dot{z}_{i}(t)=\frac{y_{i}(t)}{\tau_{i n}}-\frac{z_{i}(t)}{\tau_{R}},
$$

where $v_{i}(t)$ is the membrane potential of neuron $i$ while $y_{i}(t), z_{i}(t)$ and $x_{i}(t)=1-y_{i}(t)-z_{i}(t)$ represent the active, inactive and available fraction of resources of the corresponding synapses. The potential $v_{i}(t)$ is reset to 0 at times $t_{i}(m)$ when it reaches the threshold $v_{i}\left(t_{i}(m)\right)=1$. At $t_{i}(m)$, a spike activates a fraction $u$ of the available resources, and the activation is modeled as a spike train $S_{i}(t)=\sum_{m} \delta\left(t-t_{i}(m)\right)$. Neurons are characterized by the coupling constant $g k_{i}$, randomly extracted from the distribution $P\left(k_{i}\right) . N k_{i}$ can be interpreted as the effective number of neural synapses interacting with neuron $i$, i.e its in-degree [44]. In this framework, $k_{i}$ is the only relevant topological feature of the neural network and it justifies the DMF name. In a mean field description, the incoming synaptic current can be written as the average of the active resources $Y(t)=N^{-1} \sum_{i=1}^{N} y_{i}(t)$.

By introducing an event driven map [47], the DMF approach allows for very effective numerical simulations and it has been shown to reproduce the relevant collective dynamics for networks with large finite connectivity and metrical features [48] (see Supplemental Material (SM) [49]).

Eqs. (1-3) are characterized by three time scales: the period of the oscillating non interacting neuron $T=$ $\log (a /(a-1))$, the recovery time $\tau_{R}$ and the synaptic decay time $\tau_{i n}$. The regime $\tau_{i n} \lesssim T$ has been studied in detail in $[44,48,55,56]$, and it features a transition from a quasi-synchronous to an asynchronous phase as a function of $g$ and of the shape of $P\left(k_{i}\right)$. Here we will focus instead on the regime $\tau_{i n} \ll T \ll \tau_{R}$, setting $a=1.3, \tau_{R}=10$ and varying $\tau_{i n}$ between $10^{-1}$ and $10^{-5}$. These parameters are consistent with those selected in [46], where they have been chosen on the basis of biological motivations.

Mean Field. The presence of a further non trivial phase can be put into evidence by considering the simple in-degree distribution $P\left(k_{i}\right)=\delta\left(k_{i}-k_{0}\right)$. In this fully MF case, where all the coupling constants are equal, all neurons become completely synchronized after an initial transient state, as shown in the SM. Hence, Eqs. (1-3) reduce to the equations of a single neuron with coupling $k_{0}$ and $Y(t)=y(t)$. The dynamics can be rewritten as an event driven Poincaré map in $z_{n}$ and $y_{n}$, representing the inactive and active resources before the $n$-th synchronous spiking event (see SM):

$$
\begin{aligned}
y_{n+1}= & e^{-\frac{\Delta_{n}}{\tau_{i n}}}\left(y_{n}+u\left(1-y_{n}-z_{n}\right)\right) \\
z_{n+1}= & -e^{-\frac{\Delta_{n}}{\tau_{i n}}} \frac{y_{n}+u\left(1-y_{n}-z_{n}\right)}{1-\tau_{i n} / \tau_{R}} \\
& +e^{-\frac{\Delta_{n}}{\tau_{R}}}\left(z_{n}+\frac{y_{n}+u\left(1-y_{n}-z_{n}\right)}{1-\tau_{i n} / \tau_{R}}\right),
\end{aligned}
$$

where the time interval $\Delta_{n}$ between the $n$-th and the $(n+1)$-th spiking event is obtained from:

$$
\begin{aligned}
1= & a-e^{-\frac{\Delta_{n}}{\tau_{i n}}} \frac{g \tau_{i n} k_{0}\left(y_{n}+u\left(1-y_{n}-z_{n}\right)\right)}{1-\tau_{i n}} \\
& -e^{-\Delta_{n}}\left(a-\frac{g \tau_{i n} k_{0}\left(y_{n}+u\left(1-y_{n}-z_{n}\right)\right)}{1-\tau_{i n}}\right) .
\end{aligned}
$$

When $\tau_{\text {in }} \ll T \ll \tau_{R}$, an insight on the dynamics can be achieved by considering the opposite regimes of weak and strong interaction, i.e. when $g k_{0} Y(t)$ or $a-$ $v_{k_{0}}(t)$ are negligible in Eq. (1), respectively. In both extreme regimes, the map in Eqs. (4-6) can be solved, and it features a fixed point corresponding to a periodic solution in the continuous dynamics (see SM for details). In particular, in the weak coupling regime, the periodicity is trivially $T$, and the interaction term remains negligible if $g k_{0} \tau_{i n} \ll \tau_{R} / T$. On the other hand, if the $a-v_{k_{0}}(t)$ term can be ignored, the system displays a much faster periodicity: $T_{f}=\tau_{R} /\left(g k_{0} \tau_{i n}\right)$ and the approximations holds only if $g k_{0} \tau_{i n} \gg \tau_{R} / \tau_{i n}$.

If $\tau_{R} / T \ll g k_{0} \tau_{i n} \ll \tau_{R} / \tau_{i n}$, neither the weak nor the strong coupling conditions are satisfied, and the competition between the terms with a slow and a fast dynamics plays a non trivial role, destroying the presence of a periodic evolution. Such a behavior can be analyzed by means of the bifurcation diagram [57] of $\Delta_{n}$ as a function of $g$ at fixed $\tau_{i n}$. Fig. 1 shows the presence of a stable fixed point for small and large values of $g$, describing a slow and a fast periodic regime, respectively. For an intermediate value, a period doubling appears first; then, at $g>g^{\prime}\left(\tau_{i n}\right)$, the distribution of $\Delta_{n}$ becomes continuous. The $\Delta_{n}$ becomes again deltadistributed for $g>g^{\prime \prime}\left(\tau_{i n}\right)$. In the SM we show that for $g^{\prime}\left(\tau_{i n}\right)<g<g^{\prime \prime}\left(\tau_{i n}\right)$ the maximum Lyapunov exponent [58] becomes positive, a signature of the presence of chaos. In the fully MF system with $N$ neurons, this is an example of synchronous chaos [42, 43]. The phase diagram in Fig. 2 shows that the $\tau_{i n}$-dependence of the boundaries of the chaotic phase (squares) is consistent with the continuous lines, obtained by the weak and strong coupling limit arguments. The critical values for $g$ and $\tau_{i n}$ depend on $a$, i.e. the intrinsic period of the neuron; the chaotic dynamics is observed at higher $\tau_{\text {in }}$ by considering smaller $a$ (see SM). Taking the limit $\tau_{\text {in }} \rightarrow 0$ with $g_{\text {eff }}=g k_{0} \tau_{\text {in }}$ constant in Eqs. (4-6), one obtains a single variable map as a function of $g_{\text {eff }}, a$ and $\tau_{R}$ only, that can be studied analytically (see SM). This simpler map confirms the presence of a genuine chaotic dynamical phase.

Degree based Mean Field. Let us now focus on the multi-site DMF model with heterogeneous couplings extracted from the distribution $P\left(k_{i}\right)$. We consider a Gaussian $P\left(k_{i}\right)$ with average $\mu=0.7$ and standard deviation $\sigma=0.077$, although our results are robust for different distributions (see SM for a discussion). A relevant quantity describing the level of synchronization of the neurons 


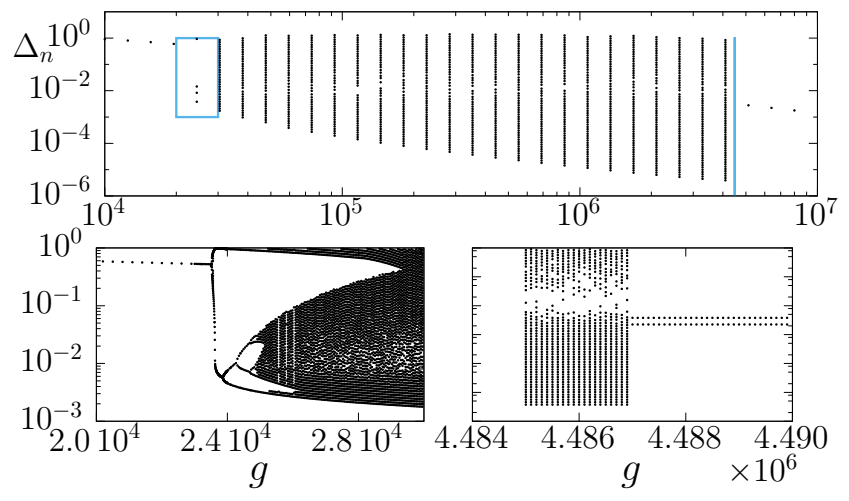

FIG. 1. Feigenbaum bifurcation diagram for the MF TUM model in Eqs. (4-5) with $\tau_{i n}=10^{-3}$. The attractor for the interspike interval of the network $\Delta_{n}$ is showed as a function of the coupling $g$. Upper panel: bifurcation diagram in the full relevant range of the parameter $g$. Lower-left panel: magnification on the period doubling cascade at the first transition. Lower-right panel: magnification on the second transition. The blue rectangles in the upper panel indicate the zooming regions of the lower panels.

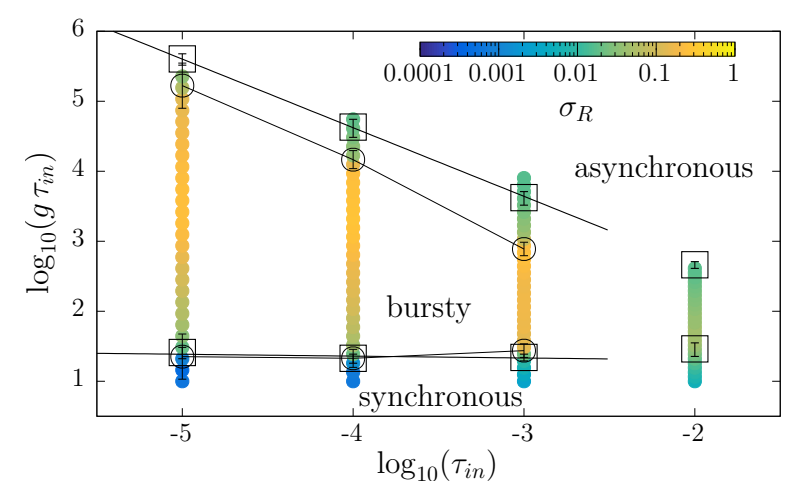

FIG. 2. (Color online) Dynamical phase diagram of the MF and DMF models in terms of the coupling constants $g$ and of the synaptic time scale $\tau_{\text {in }}$. MF model: The squares indicate the $g$ values at which the transition to chaos (along with the discontinuity of the interspike time standard deviation) takes place (see SM). The black lines are linear fits. DMF model: Each colored point corresponds to a simulation, the color code indicating $\sigma_{R}$ at the corresponding value of $\left(g, \tau_{\text {in }}\right)$. The intervals of $g$ containing the discontinuity (c.f. Fig. 3) are signaled with black circles.

is the Kuramoto parameter [59]: $R(t)=\frac{1}{N}\left|\sum_{i=1}^{N} \mathrm{e}^{\imath \phi_{i}(t)}\right|$ where $\phi_{i}(t)$ is the phase of neuron $i$ at time $t$ :

$$
\phi_{i}(t)=2 \pi \frac{t-t_{i}(m)}{t_{i}(m+1)-t_{i}(m)}
$$

where $t_{i}(m)$ is the $m$-th spike of neuron $i$ and $t \in$ $\left[t_{i}(m), t_{i}(m+1)\right]$. In Fig. 3 the time average $\langle R\rangle$ of the Kuramoto parameter and its fluctuations $\sigma_{R}$ are displayed as a function of $g$. At small couplings, $\langle R\rangle \approx 1$ and the fluctuations are small, as the systems is in a

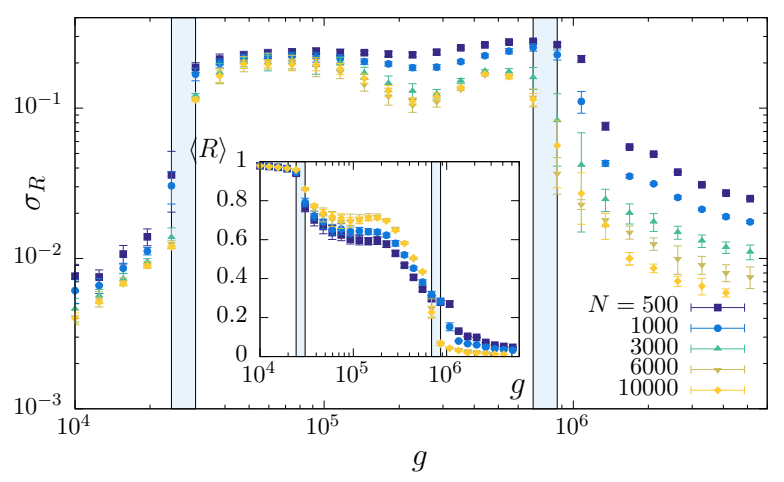

FIG. 3. (Color online) Standard deviation of the Kuramoto parameter, $\sigma_{R}$ versus $g$ for the DMF model with $\tau_{\text {in }}=10^{-3}$ and five values of $N$. In the quasi-synchronous and bursty phases the data corresponding to the two larger values of $N$ overlap within their statistical errors, indicating convergence in size, while deep in the asynchronous phase they decrease as $\sim N^{-1 / 2}$. Inset: temporal average of $R(t)$, showing that the larger sizes have attained their asymptotic value in all the phases. The vertical stripes are common to all the figures in the article and indicate the apparent discontinuity of $\langle R\rangle$ for the largest sizes.

quasi-synchronous phase. At large $g,\langle R\rangle$ becomes very small $(\langle R\rangle \rightarrow 0$ with increasing $N)$, consistently with a periodic asynchronous phase. In the irregular, bursty, regime, $\langle R\rangle$ exhibits moderate values and, more significantly, its fluctuations grow abruptly by an order of magnitude; this is a signal of a complex dynamical phase, illustrated in the raster plot in the inset of Fig. 4 (each dot corresponds to a spike of neuron $i$ at time $t$ ). The fluctuations of $\langle R\rangle$ originate from the alternations of synchronous events with asynchronous phases characterized by smaller bursts where only a subset of the neurons fires simultaneously. The main plot of Fig. 4 shows that the size $s$ of such bursts, or avalanches, is broadly distributed (see SM for a detailed definition of burst size). Interestingly, the distribution is compatible with a power law $h(s) \sim s^{-\gamma}$ followed by a bump. The power $\gamma$, close to 2 (see SM), does not depend significantly on $N$, nor on $g$ for a wide $g$-range in the bursty phase. Finally, the peaks at large $s$ in the distributions correspond to synchronous events where all neurons fire quasi-simultaneously, and their position scales with the system size.

The natural issue is the relation between the chaotic phase in the single site MF model and the burstyavalanche regime of the multi-site DMF approach. In the SM we show that also the bursty phase is characterized by a chaotic dynamics with positive Lyapunov exponents. In Fig. 2 we have superimposed the dynamical phase diagrams of the MF and DMF models. In the DMF, the transitions points (circles) are set at the $g$ intervals at which the abrupt increments of the fluctuations of the Kuramoto parameter take place (c.f. Fig. 3). In the MF case, the squares indicate the values of $g$ 


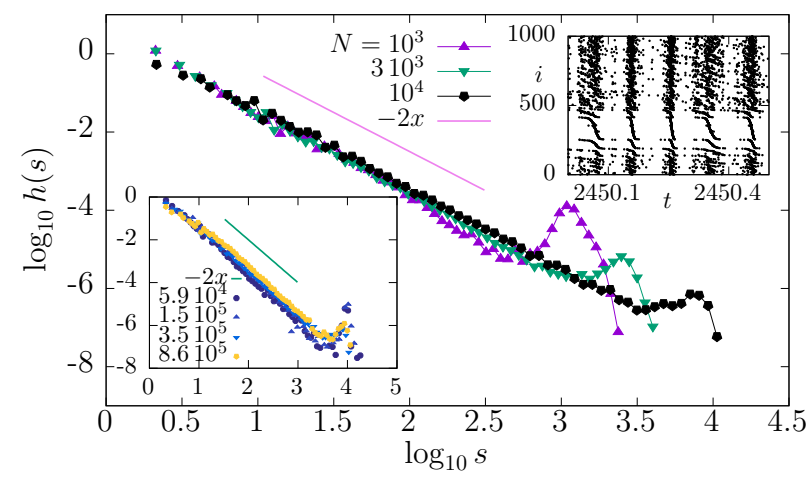

FIG. 4. (Color online) Avalanche size histogram $h(s)$ of the DMF model, $\tau_{\text {in }}=10^{-3}$ and $g=3.5 \cdot 10^{5}$ in the bursty regime, for several values of $N$. Upper inset: a fragment of the raster plot for the same system. Lower inset: $\log _{10} h(s)$ for $N=10^{4}$ and various values of $g$ across the bursty phase.

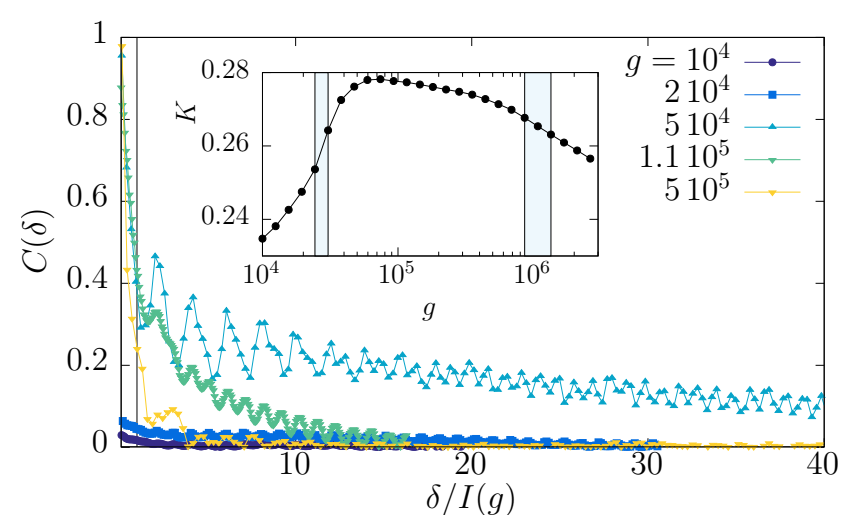

FIG. 5. (Color online) Main plot: connected correlation function $C$ as a function of the time difference $\delta$ in units of the average interspike time $I(g)$, for several values of $g$ in the DMF model with $\tau_{i n}=10^{-3}, N=800$. For values of $g$ in the bursty phase, the correlation remains high even after large time differences. Inset: Kolmogorov Complexity of the DMF model, $\tau_{\text {in }}=10^{-3}, N=10^{3}$, and the spike time differences $\Delta_{n}$ stored with 14 digits of precision.

at which the transitions to chaos occur. While the phase diagrams slightly differ, the phase diagram of the DMF model converges continuously to that of the MF model in the limit of vanishing width of the distribution $P\left(k_{i}\right)$, as illustrated in the SM. This scenario suggests that the bursty regime arises from the introduction of disorder on a system with synchronous chaos, so that neurons with different coupling $k_{i}$ do not fire simultaneously and the synchronous solution loses stability.

In the DMF model, the transition to the bursty collective behavior also corresponds to the presence of large temporal correlations. We define the time dependent complex correlation, $c(\delta, t)=$ $(1 / N) \sum_{i=1}^{N} e^{\imath \phi_{i}(t)} e^{-\imath \phi_{i}(t+\delta)}$, where $\phi_{i}(t)$ is the Kuramoto phase (7), along with the connected correlation function, $C(\delta)=\left|\langle c(\delta, t)\rangle_{t}\right|-\left|\langle c(\mathcal{T}, t)\rangle_{t}\right|$, as the temporal average $\langle\cdot\rangle_{t}$ of $c$ over a sufficiently large interval of times $t$, minus its stationary value at a sufficiently large time difference, $\delta=\mathcal{T}$ (for details at this regard see the SM section). $C(\delta)$ measures in this way the average amount of correlation between spike configurations separated by a time delay $\delta$. The quantity $C(\delta)$ (see Fig. 5, main panel) reveals the existence of large correlations for times $\delta$ much larger than the average interspike time, $I(g)$, only in the bursty regime (for $3 \cdot 10^{4} \lesssim g \lesssim 10^{5}$ at $\tau_{\text {in }}=10^{-3}$ ), while in the synchronous and asynchronous regimes, $C(\delta)$ decays faster to its asymptotic value.

Another interesting quantity in temporal series of neural firing patterns is the amount of information they can sustain. In information theory, the Kolmogorov Complexity (KC) of a data sequence determines the length of the minimum computer program generating it, hence being a measure of the sequence predictability [60]. KC has been related to the computational power of artificial neural networks [61], and used in the quantitative characterization of epileptic EEG recordings [62]. We consider the $\mathrm{KC}$ of the raster plot, interpreting it as an estimation of the amount of information that can be codified in the dynamical signal (see the details of the KC estimation in the SM section). The numerical results for the DMF model reveal that the $\mathrm{KC}$ as a function of $g$ (see the inset of Fig. 5) presents a maximum in the bursty regime (around $g \simeq 610^{4}$ for $\tau_{i n}=10^{-3}$ ).

In summary, we have reported the existence of a dynamical phase occurring in a network of purely excitatory LIF neurons connected with synaptic plasticity. This phase, identified by average statistical properties of the Kuramoto parameter, is strongly chaotic and it differs from previously known irregular phases for similar models, e.g. phases with chaotic transient dynamics [31, 63]. The chaotic phase must also be distinguished from previous irregular regimes observed in spiking neural models, namely weak chaos in purely excitatory disordered networks [64] or stable chaos in inhibitory ones [65-67]. The emergent dynamical regime occurs in a large region of the phase diagram, and it is separated by two dynamical transitions from the quasi-synchronous and asynchronous regimes. Chaos is preserved in the presence of disordered couplings. In that case, interestingly, the chaotic phase also features characteristic power law distributed avalanches. By properly defining temporal correlations and tools from information theory, we show that the additional bursty phase is strongly correlated and it carries a relevant amount of information compared to the quasisynchronous and the asynchronous phases.

We gratefully acknowledge the support of NVIDIA Corporation with the donation of the Tesla K40 GPU used for this research. We warmly thank S. di Santo, R. Livi, M. A. Muñoz and A. Politi for useful discussions. 
[1] T. P. Vogels, K. Rajan, and L. Abbott, Annu. Rev. Neurosci. 28, 357 (2005).

[2] E. Hulata, V. Volman, and E. Ben-Jacob, Nat. Comput. 4, 363 (2005).

[3] Y. Shu, A. Hasenstaub, and D. A. McCormick, Nature 423, 288 (2003).

[4] X.-J. Wang, Physiol. Rev. 90, 1195 (2010).

[5] A. Destexhe, S. W. Hughes, M. Rudolph, and V. Crunelli, Trends Neurosci. 30, 334 (2007).

[6] J. Hesse and T. Gross, Front. Syst. Neurosci. 8 (2014).

[7] M. V. Sanchez-Vives and D. A. McCormick, Nat. Neurosci. 3, 1027 (2000).

[8] L. F. Abbott and C. van Vreeswijk, Phys. Rev. E 48, 1483 (1993).

[9] C. van Vreeswijk, Phys. Rev. E 54, 5522 (1996).

[10] J. Kadmon and H. Sompolinsky, Phys. Rev. X 5, 041030 (2015).

[11] N. Brunel, J. Comput. Neurosci. 8, 183 (2000).

[12] C. Kirst, T. Geisel, and M. Timme, Phys. Rev. Lett. 102, 068101 (2009).

[13] E. Montbrió, D. Pazó, and A. Roxin, Phys. Rev. X 5, 021028 (2015).

[14] D. Hansel and G. Mato, Neural Comput. 15, 1 (2003).

[15] S. Panzeri, R. S. Petersen, S. R. Schultz, M. Lebedev, and M. E. Diamond, Neuron 29, 769 (2001).

[16] J. F. Mejias, H. J. Kappen, and J. J. Torres, PLoS ONE 5, e13651 (2010).

[17] R. Livi, Chaos Soliton Fract. 55, 60 (2013).

[18] B. D. Burns and A. C. Webb, Proc. R. Soc. B 194, 211 (1976).

[19] M. London, A. Roth, L. Beeren, M. Hausser, and P. E. Latham, Nature 466, 123 (2010).

[20] J. M. Beggs and D. Plenz, J. Neurosci. 23, 11167 (2003).

[21] R. Segev, Y. Shapira, M. Benveniste, and E. Ben-Jacob, Phys. Rev. E 64, 011920 (2001).

[22] T. Petermann, T. C. Thiagarajan, M. A. Lebedev, M. A. Nicolelis, D. R. Chialvo, and D. Plenz, Proc. Natl. Acad. Sci. U.S.A. 106, 15921 (2009).

[23] S. El Boustani, O. Marre, S. Béhuret, P. Baudot, P. Yger, T. Bal, A. Destexhe, and Y. Frégnac, PLoS Comput. Biol. 5, e1000519 (2009).

[24] O. Kinouchi and M. Copelli, Nat. Phys. 2, 348 (2006).

[25] S. Ostojic, Nat. Neurosci. 17, 594 (2014).

[26] M. Monteforte and F. Wolf, Phys. Rev. X 2, 041007 (2012).

[27] D. R. Chialvo, Nat. Phys. 6, 744 (2010).

[28] P. Massobrio, L. de Arcangelis, V. Pasquale, H. J. Jensen, and D. Plenz, Front. Syst. Neurosci. 9, 22 (2015).

[29] D. J. Amit and N. Brunel, Cereb. Cortex 7, 237 (1997).

[30] C. van Vreeswijk and H. Sompolinsky, Science 274, 1724 (1996).

[31] J. M. Cortes, M. Desroches, S. Rodrigues, R. Veltz, M. A. Muñoz, and T. J. Sejnowski, Proc. Natl. Acad. Sci. U.S.A. 110, 16610 (2013).

[32] A. Levina, J. M. Herrmann, and T. Geisel, Nat. Phys. 3, 857 (2007).

[33] J. A. Bonachela and M. A. Muñoz, J. Stat. Mech. P09009 (2009).

[34] J. A. Bonachela, S. de Franciscis, J. J. Torres, and M. A. Muñoz, J. Stat. Mech. P02015 (2010).

[35] D. Millman, S. Mihalas, A. Kirkwood, and E. Niebur,
Nat. Phys. 6, 801 (2010).

[36] V. Volman, I. Baruchi, E. Persi, and E. Ben-Jacob, Physica A 335, 249 (2004).

[37] L. de Arcangelis, C. Perrone-Capano, and H. J. Herrmann, Phys. Rev. Lett. 96, 028107 (2006).

[38] S. di Santo, R. Burioni, A. Vezzani, and M. A. Muñoz, Phys. Rev. Lett. 116, 240601 (2016).

[39] F. Lombardi, H. J. Herrmann, C. Perrone-Capano, D. Plenz, and L. de Arcangelis, Phys. Rev. Lett. 108, 228703 (2012).

[40] J. Touboul and A. Destexhe, PLoS ONE 5, e8982 (2010).

[41] J. Touboul and A. Destexhe, arXiv:1503.08033v4.

[42] M. Ding, and W. Yang, Phys.Rev. E 56, 4009 (1997).

[43] J.F. Heagy, T.L. Carroll, and L.M. Pecora, Phys.Rev. E 50, 1874 (1994).

[44] R. Burioni, M. Casartelli, M. di Volo, R. Livi, and A. Vezzani, Sci. Rep. 4, 4336 (2014).

[45] E. M. Izhikevich, IEEE Trans. Neural Networ. 15, 1063 (2004).

[46] M. Tsodyks, A. Uziel, and H. Markram, J. Neurosci. 20, 825 (2000).

[47] R. Brette, Neural Comput. 19, 2604 (2007).

[48] M. di Volo, R. Burioni, M. Casartelli, R. Livi, and A. Vezzani, Phys. Rev. E 90, 022811 (2014).

[49] Supplemental Material includes Refs. [50-54].

[50] G. Benettin, L. Galgani, A. Giorgilli, and J. M. Strelcyn, Meccanica 15, 9 (1980).

[51] I. Shimada, and T. Nagashima, Progr. Theor. Phys. 61, 1605 (1979) .

[52] M. Tsodyks, and H. Markram, PNAS 94, 719-23 (1997).

[53] M. Tsodyks, K. Pawelzick, and H. Markram, Neural Comput. 15, 821-35 (1998).

[54] A. Kaitchenko, in Canadian Conference on Electrical and Computer Engineering, (IEEE, 4, pg. 2255 2004).

[55] M. di Volo, R. Livi, S. Luccioli, A. Politi, and A. Torcini, Phys. Rev. E 87, 032801 (2013).

[56] R. Burioni, S. di Santo, M. di Volo, and A. Vezzani, Phys. Rev. E 90, 042918 (2014).

[57] See e.g.: E. Ott Chaos in dynamical systems. (Cambridge University Press, Cambridge, 2001).

[58] G. Benettin, L. Galgani, A. Giorgilli, and J. M. Strelcyn, Meccanica 15, 21 (1980).

[59] J. A. Acebrón, L. L. Bonilla, C. J. Pérez Vicente, F. Ritort, and R. Spigler, Rev. Mod. Phys. 77, 137 (2005).

[60] M. Li and P. Vitányi, An introduction to Kolmogorov complexity and its applications (Springer, New York, 1993).

[61] J. L. Balcazar, R. Gavalda, and H. T. Siegelmann, IEEE Trans. Inf. Theory 43, 1175 (1997).

[62] A. Petrosian, in Proceedings of the Eighth IEEE Symposium on Computer-Based Medical Systems, Lubbock, Texas, 1995 (1995) p. 212.

[63] R. Zillmer, R. Livi, A. Politi, and A. Torcini, Phys. Rev. E 74, 036203 (2006).

[64] S. Olmi, R. Livi, A. Politi, and A. Torcini, Phys. Rev. E 81, 046119 (2010).

[65] R. Zillmer, N. Brunel, and D. Hansel, Phys. Rev. E 79, 031909 (2009).

[66] E. Ullner and A. Politi, Phys. Rev. X 6, 011015 (2016).

[67] D. Pazó and E. Montbrió, Phys. Rev. Lett. 116, 238101 (2016). 


\title{
Chaos and correlated avalanches in neural networks with synaptic plasticity
}

\section{(Supplemental material)}

\author{
Fabrizio Pittorino, ${ }^{1,2}$ Miguel Ibáñez-Berganza, ${ }^{2}$ Matteo di \\ Volo ${ }^{3}$ Alessandro Vezzani, ${ }^{4,1, *}$ and Raffaella Burioni ${ }^{1,2}$ \\ ${ }^{1}$ Dipartimento di Scienze Matematiche, \\ Fisiche e Informatiche, Università di Parma, \\ via G.P. Usberti, 7/A - 43124, Parma, Italy \\ ${ }^{2}$ INFN, Gruppo Collegato di Parma, via G.P. Usberti, 7/A - 43124, Parma, Italy \\ ${ }^{3}$ Group for Neural Theory, Laboratoire de Neurosciences Cognitives, \\ INSERM U960, Ecole Normale Superieure, Paris, France \\ ${ }^{4}$ IMEM-CNR, Parco Area delle Scienze, 37/A-43124 Parma, Italy
}




\section{CONTENTS}

I. The Leaky integrate-and-fire (LIF) model with TUM synaptic plasticity

$\begin{array}{ll}\text { II. Mean Field } & 5\end{array}$

A. Competition of time scales in the Mean Field model 5

$\begin{array}{ll}\text { B. Weak coupling } & 6\end{array}$

$\begin{array}{ll}\text { C. Strong coupling } & 7\end{array}$

D. Chaos in the Mean Field model $\quad 7$

E. The limit $\tau_{i n} \rightarrow 0 \quad 9$

III. Degree based Mean Field 13

A. Event driven dynamics simulation protocol 13

B. Global Synaptic field $\quad 14$

C. Fluctuations of the interspike interval, Kuramoto parameter and global field 15

$\begin{array}{ll}\text { D. Lyapunov exponents } & 17\end{array}$

E. Comparison between the DMF and MF models phase diagrams 18

F. Robustness with respect to the shape of the distribution $P\left(k_{i}\right) \quad 19$

G. Robustness with respect to the single neuron time scale 20

H. Degree based Mean Field and finite connectivity systems 21

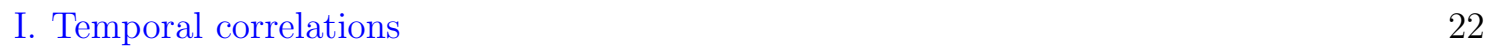

J. Kolmogorov Complexity 24

K. Avalanche size distribution 24

$\begin{array}{ll}\text { References } & 25\end{array}$

\section{THE LEAKY INTEGRATE-AND-FIRE (LIF) MODEL WITH TUM SYNAPTIC PLASTICITY}

Let us consider a system of $N$ neurons and call $k_{i}$ the coupling of neuron $i$, where $k_{i}$ is extracted from the distribution $P\left(k_{i}\right)^{1}$. The $N k_{i}$ can also be considered as the in-degree of neuron $i$, so that $P\left(k_{i}\right)$ is a degree distribution. The equations of motion of the Degree based Mean Field (DMF) 
TUM model are:

$$
\begin{aligned}
& \dot{v}_{i}(t)=a-v_{i}(t)+g k_{i} \frac{1}{N} \sum_{j=1}^{N} y_{j}(t) \\
& \dot{y}_{i}(t)=-\frac{y_{i}(t)}{\tau_{i n}}+u\left(1-y_{i}(t)-z_{i}(t)\right) S_{i}(t) \\
& \dot{z}_{i}(t)=\frac{y_{i}(t)}{\tau_{\text {in }}}-\frac{z_{i}(t)}{\tau_{R}}
\end{aligned}
$$

where $v_{i}(t)$ is reset to 0 at the firing events $t_{i}(m)$ that correspond to $v_{i}\left(t_{i}(m)\right)=1$, and $S_{i}(t)=$ $\sum_{m} \delta\left(t-t_{i}(m)\right)$ is the spike train of neuron $i$.

Eq.s (1) can be solved by an event driven map ${ }^{2}$. We denote with $v_{i, n}, y_{i, n}$ and $z_{i, n}$ the value of $v_{i}\left(t_{n}\right), y_{i}\left(t_{n}\right)$ and $z_{i}\left(t_{n}\right)$ immediately before the $n$-th firing event and with $\Delta_{n}=t_{n+1}-t_{n}$ the time interval between the firing events $\left(t_{i}(m)\right.$ denotes firing times of neuron $i$ while $t_{n}$ represents the sequence of firing times of the network, independently of the firing neuron). The evolution of the discrete variables then reads:

$$
\begin{aligned}
& v_{i, n+1}=v_{i, n} e^{-\Delta_{n}}+a\left(1-e^{-\Delta_{n}}\right)+\frac{g k_{i} \tau_{\text {in }}}{\tau_{\text {in }}-1}\left(e^{-\Delta_{n} / \tau_{\text {in }}}-e^{-\Delta_{n}}\right) \frac{1}{N} \sum_{j=1}^{N} y_{j, n} \\
& y_{i, n+1}=\left(y_{i, n}+\delta_{i, s_{n+1}} u\left(1-y_{i, n}-z_{i, n}\right)\right) e^{-\Delta_{n} / \tau_{\mathrm{in}}} \\
& z_{i, n+1}=z_{i, n} e^{-\Delta_{n} / \tau_{R}}+\frac{\tau_{\mathrm{R}}}{\tau_{\mathrm{R}}-\tau_{\mathrm{in}}}\left(y_{i, n}+\delta_{i, s_{n+1}} u\left(1-y_{i, n}-z_{i, n}\right)\right)\left(e^{-\Delta_{n} / \tau_{\mathrm{R}}}-e^{-\Delta_{n} / \tau_{\mathrm{in}}}\right),
\end{aligned}
$$

where $\delta_{i, j}$ is the Kronecker delta and $s_{n}$ is the neuron that fires at time $t_{n}$. To implement the reset rule on the potential, for neuron $s_{n+1}$ that fires at $t_{n+1}$ the first Eq. (2) is replaced by:

$$
1=v_{s_{n+1}, n} e^{-\Delta_{n}}+a\left(1-e^{-\Delta_{n}}\right)+g k_{i} \tau_{\text {in }} \frac{e^{-\Delta_{n} / \tau_{\mathrm{in}}}-e^{-\Delta_{n}}}{\tau_{\text {in }}-1} \frac{1}{N} \sum_{j=1}^{N}\left(y_{j, n}+\delta_{j, s_{n}} u\left(1-y_{j, n}-z_{j, n}\right)\right)
$$

and $v_{s_{n+1}, n+1}=0$. Notice that Eq. (3) allows to calculate $\Delta_{n}$ and that the firing neuron is identified by finding the value of $s_{n+1}$ that provides the minimum value of $\Delta_{n}$ in Eq. (3). 

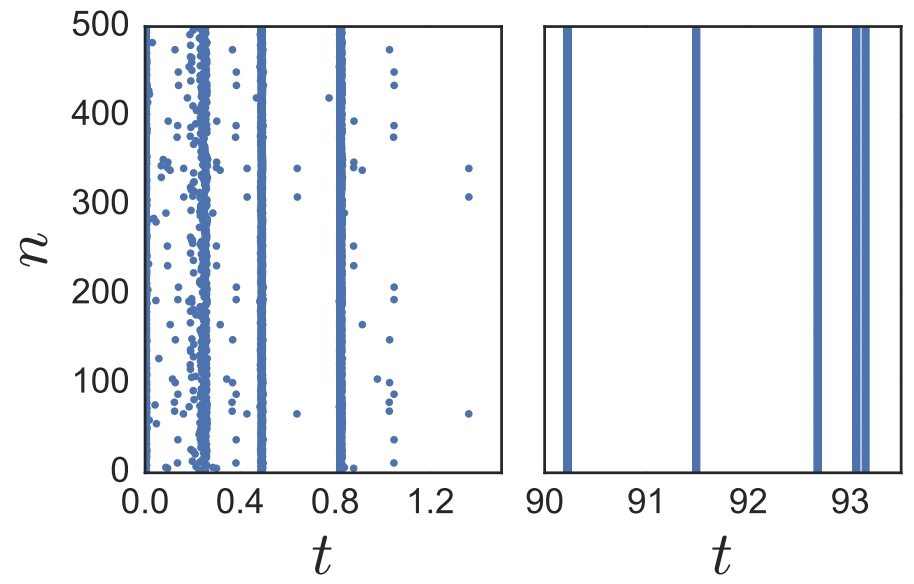

FIG. 1. Raster plot of a fully MF system with $k_{i}=k_{0}=0.7, g=10^{6} \tau_{\text {in }}=10^{-3}$ each dot represents the firing event of neuron $n$. Left: short time evolution. Right: in the long time evolution the system reaches a synchronous but chaotic state.

Let us consider the Mean Field (MF) case where $k_{i}=k_{0}$ for all sites. In Fig. 1 we report a raster plot showing that the system converges, after a short transient, to a synchronous state where all neurons fire simultaneously. This had already been observed in a parameter setup showing periodic synchronous dynamics ${ }^{3}$. Nevertheless, in the present case the synchronous solution shows an irregular chaotic dynamics, indicating our system as an example of synchronous chaos ${ }^{4,5}$. Since each neuron displays the same dynamical evolution, the MF equations reduce to the dynamics of a single neuron representing the entire system. We can consequently drop the index $i$ and obtain:

$$
\begin{aligned}
& \dot{v}(t)=a-v(t)+g k_{0} y(t) \\
& \dot{y}(t)=-\frac{y(t)}{\tau_{\text {in }}}+u(1-y(t)-z(t)) S(t) \\
& \dot{z}(t)=\frac{y(t)}{\tau_{\text {in }}}-\frac{z_{i}(t)}{\tau_{R}} .
\end{aligned}
$$

The corresponding event driven map reads

$$
\begin{aligned}
1 & =a\left(1-e^{-\Delta_{n}}\right)+\frac{g k_{0} \tau_{\text {in }}}{\tau_{\text {in }}-1}\left(e^{-\Delta_{n} / \tau_{\text {in }}}-e^{-\Delta_{n}}\right)\left(y_{n}+u\left(1-y_{n}-z_{n}\right)\right) \\
y_{n+1} & =\left(y_{n}+u\left(1-y_{n}-z_{n}\right)\right) e^{-\Delta_{n} / \tau_{\text {in }}} \\
z_{n+1} & =z_{n} e^{-\Delta_{n} / \tau_{R}}+\frac{\tau_{\mathrm{R}}}{\tau_{\mathrm{R}}-\tau_{\text {in }}}\left(y_{n}+u\left(1-y_{n}-z_{n}\right)\right)\left(e^{-\Delta_{n} / \tau_{\mathrm{R}}}-e^{-\Delta_{n} / \tau_{\mathrm{in}}}\right),
\end{aligned}
$$

where we take into account that the single neuron fires at each event, and therefore we use Eq. (3) with $v_{n}=0$. In practice, the first of Eq.s (5) is used to obtain the firing time intervals $\Delta_{n}$ while the other two equations represent a discrete two dimensional map for the variables $y_{n}$ and $z_{n}$. 


\section{MEAN FIELD}

\section{A. Competition of time scales in the Mean Field model}

The single neuron MF Eq.s $(4,5)$ become particularly simple in the weak coupling and strong coupling regimes. In the former, we can neglect $g k_{0} y(t)$ in Eq.s (4) while in the latter we neglect $a-v(t)$. In both limits, the map (5) has a fixed point, characterized by a slow and a fast periodicity respectively.

Let us first discuss some general property of the fixed point. We consider the regime $T=$ $\ln (a /(a-1)) \sim O(1)$ and $\tau_{\text {in }} \ll T \ll \tau_{R}$, so that the event driven dynamics (5) of the MF model becomes:

$$
\begin{aligned}
1 & =a-g \tau_{i n} k_{0} y_{n+1}-e^{-\Delta_{n}}\left(a-g \tau_{i n} k_{0}\left(y_{n}+u\left(1-y_{n}-z_{n}\right)\right)\right) \\
y_{n+1} & =e^{-\frac{\Delta_{n}}{\tau_{i n}}}\left(y_{n}+u\left(1-y_{n}-z_{n}\right)\right) \\
z_{n+1} & =-y_{n+1}+e^{-\frac{\Delta_{n}}{\tau_{R}}}\left(y_{n}+z_{n}+u\left(1-y_{n}-z_{n}\right)\right) .
\end{aligned}
$$

We denote with $\left(x^{*}, y^{*}\right)$ the fixed point of the map (6-8) and with $\Delta^{*}$ the relevant interspike interval, i.e. Eq.s (6-8) are satisfied by $y_{n+1}=y_{n}=y^{*}, x_{n+1}=x_{n}=x^{*}$ and $\Delta_{n+1}=\Delta_{n}=\Delta^{*}$.

Eq.s (7-8) hold both in the strong and in the weak coupling regimes and they describe a general property of the fixed point. In particular, considering the variable $w^{*}=y^{*}+z^{*}$, Eq. (8) reads:

$$
w^{*}=e^{-\frac{\Delta^{*}}{\tau_{R}}}\left(w^{*}+u\left(1-w^{*}\right)\right)
$$

and:

$$
w^{*}=u \frac{e^{-\frac{\Delta^{*}}{\tau_{R}}}}{1-(1-u) e^{-\frac{t^{*}}{\tau_{R}}}} \simeq 1-\frac{\Delta^{*}}{u \tau_{R}},
$$

where in the last step we used our assumption $\Delta^{*} \ll \tau_{R}$. Inserting Eq. (10) in Eq. (7), the equation for the fixed point of $y^{*}$ reads:

$$
y^{*}=e^{-\frac{\Delta^{*}}{\tau_{i n}}} \frac{\Delta^{*}}{\tau_{R}} \frac{1}{1-e^{-\frac{\Delta^{*}}{\tau_{i n}}}} .
$$

Let us come back to the differential Eq.s (4) and consider the periodic solution corresponding to the fixed point of the map. Since $y^{*}$ represent the value of $y(t)$ immediately before a firing event, if we set the time axes so that the firing events occur at $t=0, \Delta^{*}, 2 \Delta^{*} \ldots$, for $0<t<\Delta^{*}$ we have that:

$$
y(t)=\frac{\Delta^{*}}{\tau_{R}} \frac{e^{-\frac{t}{\tau_{i n}}}}{1-e^{-\frac{\Delta^{*}}{\tau_{i n}}}} .
$$


We remark that, at the fixed point, the above dynamical evolution holds for any value of the coupling constant. However, the value of $\Delta^{*}$ is determined by Eq. (6) which explicitly depends on $g$. In particular, in the strong coupling regime Eq. (6) reduces to:

$$
1=-g \tau_{i n} k_{0} y_{n+1}+g \tau_{i n} k_{0}\left(y_{n}+u\left(1-y_{n}-z_{n}\right)\right) \text {. }
$$

Imposing $y^{*}=y_{n+1}=y_{n}$ and $z^{*}=z_{n+1}=z_{n}$ we get $1=g \tau_{i n} k_{0} u\left(1-y^{*}-z^{*}\right)$, which gives

$$
w^{*}=1-\frac{1}{u k_{0} g \tau_{i n}} .
$$

Comparing Eq. (10) with Eq. (14) we get:

$$
\Delta^{*}=\Delta_{S}^{*} \simeq \frac{\tau_{R}}{g \tau_{i n} k_{0}} .
$$

On the other hand, in the weak coupling regime, we obviously obtain $\Delta^{*}=\Delta_{W}^{*}=\ln \left(\frac{a}{a-1}\right) \simeq O(1)$.

We now plug (12) into the first of Eq.s (4), describing the evolution of the membrane potentials, and we verify under which conditions the periodicity of the weak and strong coupling regime remains unperturbed. In particular, for $0<t<\Delta^{*}$, we have that $v(t)$ satisfies the equation:

$$
\dot{v}(t)=a-v(t)+\frac{g k_{0} \Delta^{*}}{\tau_{R}} \frac{e^{-\frac{t}{\tau_{i n}}}}{1-e^{-\frac{\Delta^{*}}{\tau_{i n}}}} .
$$

Solving the differential equation and imposing $\tau_{\text {in }} \ll 1$, we get:

$$
v(t)=a\left(1-e^{-t}\right)+\frac{g \tau_{i n} k_{0} \Delta^{*}}{\tau_{R}} \frac{1}{1-e^{-\Delta^{*} / \tau_{i n}}}\left(e^{-t}-e^{-\frac{t}{\tau_{i n}}}\right) .
$$

Now we need to verify under which conditions on $g$, in the weak and strong coupling regimes, the solution of $v\left(t^{*}\right)=1$ remains close to $t^{*}=\Delta_{W}^{*}$ and to $t^{*}=\Delta_{S}^{*}$, respectively.

\section{B. Weak coupling}

For small $g$, we have that the periodicity $\Delta_{W}^{*}$ and the evolution time are much larger than $\tau_{i n}$, therefore Eq. (17) can be written as:

$$
v(t) \simeq a\left(1-e^{-t}\right)+\frac{g \tau_{i n} k_{0} \Delta_{W}^{*}}{\tau_{R}} e^{-t}
$$

Solving $v\left(t^{*}\right)=1$ we get

$$
t^{*} \simeq \ln \left(\frac{a-g \tau_{i n} k_{0} \Delta_{W}^{*} / \tau_{R}}{a-1}\right)
$$

and then $t^{*} \cong \Delta_{W}^{*}=\ln (a /(a-1))$ only if $\frac{g \tau_{i n} k_{0} \Delta^{*}}{\tau_{R}} \ll 1$, i.e. the periodicity of the weak coupling regime is preserved if $g \ll g_{c}^{\text {weak }}$ with:

$$
g_{c}^{\text {weak }}=\frac{\tau_{R}}{\tau_{i n} k_{0} \ln \left(\frac{a}{a-1}\right)} .
$$




\section{Strong coupling}

In this case we have that the period $\Delta_{S}^{*}$ and the evolution time $t$ are much smaller than $T \simeq O(1)$. Therefore, we can approximate Eq. (17), so that:

$$
v(t) \simeq a t+\frac{1-e^{-\frac{t}{\tau_{i n}}}}{1-e^{-\frac{\Delta_{S}^{*}}{\tau_{i n}}}} .
$$

Let us discuss Eq. (21) considering the two opposite regimes $\Delta_{S}^{*} \ll \tau_{\text {in }}$ and $\Delta_{S}^{*} \gg \tau_{\text {in }}$. For $\Delta_{S}^{*} \ll \tau_{\text {in }}$ also $t \ll \tau_{\text {in }}$ and we can expand Eq. (21) obtaining:

$$
v(t) \simeq a t+\frac{t}{\Delta_{S}^{*}}
$$

and since $\Delta_{S}^{*} \ll \tau_{i n} \ll a^{-1}$, the solution of $v\left(t^{*}\right)=1$ is $t^{*} \simeq \Delta_{S}^{*}$. In the opposite limit, i.e. $\Delta_{S}^{*} \gg \tau_{i n}$, we have $e^{-\Delta_{S}^{*} / \tau_{i n}} \simeq 0$ and Eq. (21) can be approximated as:

$$
v(t) \simeq a t+1-e^{-\frac{t}{\tau_{i n}}}
$$

Now $v\left(t^{*}\right)=1$ implies that $a t^{*} \simeq e^{-\frac{t^{*}}{\tau_{i n}}}$ so that the firing time is of the same order of magnitude of $\tau_{\text {in }}$; this means that $t^{*} \simeq \tau_{\text {in }} \ll \Delta_{S}^{*}$. Therefore, in the full dynamics we obtain a self consistent solution $t^{*} \simeq \Delta_{S}^{*}$ which can be considered a perturbation of the strong coupling periodic evolution only for the case $\Delta_{S}^{*} \ll \tau_{i n}$, i.e. only if $g \gg g_{c}^{\text {strong }}$ with

$$
g_{c}^{\text {strong }}=\frac{\tau_{R}}{k_{0} \tau_{\text {in }}^{2}} \text {. }
$$

\section{Chaos in the Mean Field model}

According to the above discussion, in the MF TUM model we have, for small enough $\tau_{i n}$, $g_{c}^{\text {weak }} \ll g_{c}^{\text {strong }}$. We therefore expect three different regimes: for small and large values of $g$ a slow and a fast periodic dynamic should be respectively present, while in an intermediate wide range of the synaptic couplings $g_{c}^{\text {weak }} \ll g \ll g_{c}^{\text {strong }}$ the competition of the two mechanisms could give rise to a complex dynamics. We show in Fig. 2, for the MF model, the bifurcation diagrams of the temporal difference $\Delta_{n} \equiv t_{n+1}-t_{n}$ between two consecutive spikes of the network as a function of $g$ for different values of $\tau_{\text {in }}$, and the analogous values of the coefficient of variation, defined by the standard deviation of the interspike interval $\Delta_{n}$ over its mean: $\frac{\sigma_{\Delta_{n}}}{\left\langle\Delta_{n}\right\rangle}$. 

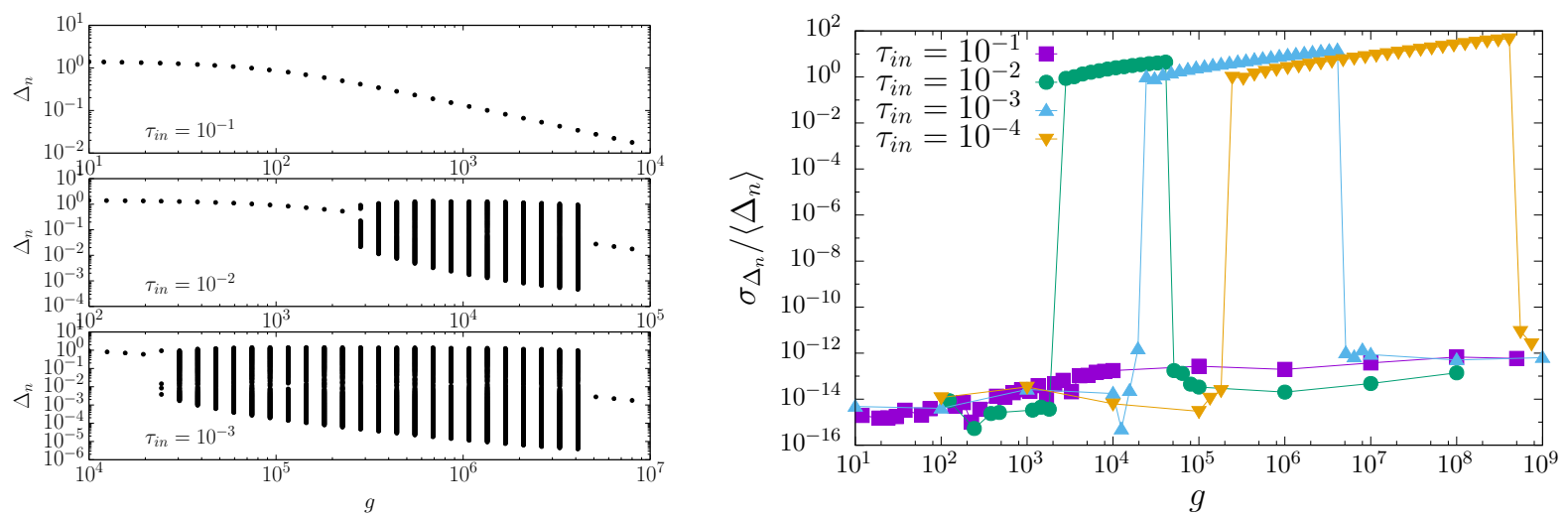

FIG. 2. Left: Feigenbaum bifurcation diagram for the MF TUM model with $\tau_{i n} \neq 0$. The attractor for the interspike interval $\Delta_{n}$ is showed as a function of the bifurcation parameter, i.e. the coupling constant $g$. The bifurcation diagram is shown for decreasing values of the synaptic time scale $\tau_{i n}$ from the top panel to the lower $\left(\tau_{i n}=10^{-1,-2,-3}\right.$ respectively) in order to show the appearance and growth of the chaotic regime in the parameter space (notice that the $\mathrm{x}$-axis range is different for each panel). Right: coefficient of variation of the interspike interval, $\frac{\sigma_{\Delta_{n}}}{\left\langle\Delta_{n}\right\rangle}$, for several values of $\tau_{i n}$. This quantity is of order 1 in the chaotic regime only.

The calculation of the Lyapunov exponents (via the Gram-Schmidt scheme ${ }^{6,7}$ ) confirms the presence of the chaotic regime in the range of the parameter $g$ between the two bifurcations, as shown in Fig. 3. Analogous figures can be obtained for other values of $\tau_{i n}$. When we consider a system of $N$ neurons coupled with the same constant $k=k_{0}$ this chaotic phase correspond to a regime of synchronous chaos ${ }^{4,5}$, see Fig. 1 for a typical raster plot. 


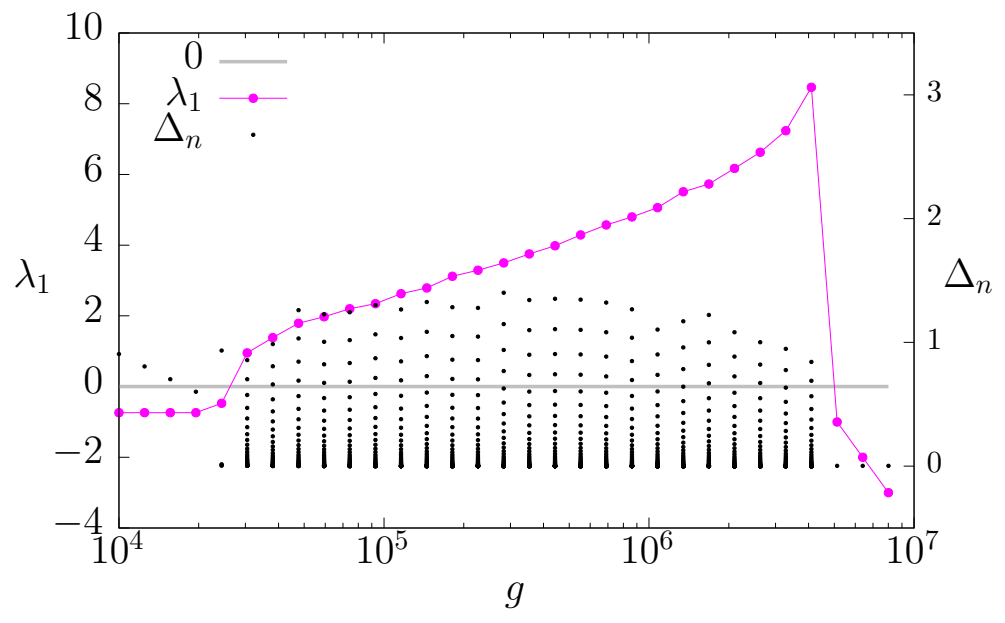

FIG. 3. First Lyapunov exponent $\lambda_{1}$ (in red) as a function of $g$ for the MF TUM model with $\tau_{i n}=10^{-3}$. In black, for comparison: bifurcation diagram as a function of $g$ for the same model (right x-axis). The sign of the first Lyapunov exponent $\lambda_{1}$ confirms a chaotic regime $\left(\lambda_{1}>0\right)$ between the synchronous-bursty and bursty-asynchronous transitions outside this region $\left(\lambda_{1}<0\right)$.

\section{E. The limit $\tau_{i n} \rightarrow 0$}

We now consider the MF dynamical Eq.s (5) in the limit $\tau_{i n} \rightarrow 0$ and $g \rightarrow \infty$ with $g \tau_{\text {in }}$ constant, so that the new effective coupling constant is defined by $g_{\text {eff }}=k_{0} g \tau_{\text {in }}$ (without loss of generality we consider $k_{0}=1$ in the rest of this section). Let us reintroduce $x=1-y-z$ and $x_{n}=1-y_{n}-z_{n}$ where $x_{n}$ are the available resources before the $n$-th firing event. If $g_{\text {eff }}\left(y_{n}+u x_{n}\right)<1$ the evolution time $\Delta_{n}$ after the $n$-th firing event is larger than zero and, in particular, from the first of Eq.s (5) we obtain:

$$
\Delta_{n}=\ln \left(\frac{a-1}{a-g_{\mathrm{eff}}\left(y_{n}+u x_{n}\right)}\right)>0 .
$$

Then, taking the limit $\tau_{i n} \rightarrow 0$ in Eq.s (5) we get $y_{n+1}=0$ and

$$
x_{n+1}=1+\left(x_{n}(1-u)-1\right) e^{-\Delta_{n} / \tau_{\mathrm{R}}}
$$

which is a discrete map counterpart of the differential equation:

$$
\dot{x}(t)=\frac{1-x(t)}{\tau_{R}}-u x(t) \sum_{m} \delta\left(t-t_{n+1}(m)\right) .
$$

Therefore, if $g_{\text {eff }}\left(y_{n}+u x_{n}\right)<1$, we reduce to a single variable map for $x_{n}$, obtained inserting the expression (25) for $\Delta_{n}$ into Eq. (26). 
On the other hand, if $g_{\text {eff }}\left(y_{n}+u x_{n}\right)>1$ the solution of the first Eq. (5) can be obtained letting also $\Delta_{n} \rightarrow 0$ with $\frac{\Delta_{n}}{\tau_{\text {in }}}$ constant, so we get

$$
1=-g_{\mathrm{eff}}\left(e^{-\Delta_{n} / \tau_{\mathrm{in}}}-1\right)\left(y_{n}+u\left(1-y_{n}-z_{n}\right)\right)
$$

or:

$$
e^{-\Delta_{n} / \tau_{\mathrm{in}}}=\frac{g_{\mathrm{eff}}\left(y_{n}+u x_{n}\right)-1}{g_{\mathrm{eff}}\left(y_{n}+u x_{n}\right)} .
$$

Plugging (29) into the second of (5) we have:

$$
y_{n+1}=y_{n}+u x_{n}-\frac{1}{g_{\mathrm{eff}}} .
$$

Taking the limits $\tau_{\text {in }} \rightarrow 0$ and $\Delta_{n} \rightarrow 0$ in the third of (5):

$$
z_{n+1}=-y_{n+1}+\left(z_{n}+y_{n}+u\left(1-y_{n}-z_{n}\right)\right)
$$

i.e.

$$
x_{n+1}=x_{n}(1-u)
$$

as it is expected in an instantaneous firing event.

In conclusion, if $g_{\text {eff }}\left(y_{n}+u x_{n}\right)<1$ the evolution time $\Delta_{n}$ is given by (25), $y_{n+1}=0$ and $x_{n+1}$ is given by Eq. (26). If $g_{\mathrm{eff}}\left(y_{n}+u x_{n}\right)>1$ the evolution is instantaneous, $\Delta_{n}=0$ and the maps for $x_{n}$ and $y_{n}$ are described by Eq.s (30) and (32).

An analogous limit $\tau_{i n} \rightarrow 0$ can be performed also on the DMF equations obtaining for the dynamical variables $v_{i}(t)$ and $x_{i}(t)$ a set of equations similar to the TUM model described in ${ }^{8}$ :

$$
\begin{aligned}
& \dot{v}_{i}(t)=a-v_{i}(t)+g \tau_{i n} k_{i} \frac{1}{N} \sum_{j=1}^{N} x_{j}(t) S_{j}(t) \\
& \dot{x}_{i}(t)=\frac{1-x_{i}(t)}{\tau_{R}}-u x_{i}(t) S_{i}(t)
\end{aligned}
$$

where $g \tau_{i n}$ is the new effective coupling constant. Moreover, also in this case simultaneous firing events are possible. In particular, if neuron $j$ fires at time $t_{m}$ and $v_{i}\left(t_{m}\right)+u N^{-1} x_{j}\left(t_{m}\right)>1$ also neuron $i$ fires at $t_{m}$ and $v_{i}\left(t_{m}\right)$ is set to $v_{i}\left(t_{m}\right)+u N^{-1} x_{j}\left(t_{m}\right)-1$. We remark that also in the DMF case, reintroducing the potential variable $v_{n+1}=g_{\text {eff }}\left(y_{n}+u x_{n}\right)$ in Eq.s $(30,32)$, we can eliminate $y_{n}$ obtaining $v_{n+1}=v_{n}+u g_{\text {eff }} x_{n}-1$, i.e. the natural equation for a neuron that receive an input $u g_{\text {eff }} x_{n}$ and simultaneously fires decreasing by one unit.

In the DMF case, the dynamical description can be further summarized introducing two new indexes $m$ and $p$, labeling the firing events. The label $m$ increases of one unit each time there is a 
firing event such that $\Delta_{n}>0$ and $p=1 \ldots, p_{m}$ labels the different simultaneous firing events. In this way we replace the index $n$ with the couple $(m, p)$ : if in the firing event $\Delta_{n}=0, m$ remains fixed and $p$ increases by one, while if $\Delta_{n}>0, m$ increases by one and $p$ is reset to $1\left(p_{m}\right.$ is the value of $p$ for which $m$ is increased to $m+1$ and $p$ is reset). With this new labels for the events, we denote $x_{(p, k)}=x_{n}, y_{(p, k)}=y_{n}$ and so on.

For a given value of $m$, from $p=1$ to $p=p_{m}$, Eq.s (30) and (32) can be easily evaluated obtaining:

$$
x_{(m, p)}=x_{(m, 1)}(1-u)^{p-1}
$$

and

$$
y_{(m, p)}=\left(1-(1-u)^{p-1}\right) x_{(m, 1)}-\frac{p-1}{g_{\mathrm{eff}}}
$$

where Eq. (36) can be verified recursively taking into account that $y_{(m, 1)}=0$. The number of simultaneous firings $p_{m}$ can be evaluated by verifying if $g_{\mathrm{eff}}\left(y_{(p, k)}+u x_{(p, k)}\right)<1$, in particular from Eq.s (35) and (36) we obtain that $p_{m}$ is the smallest positive integer such that:

$$
g x_{(m, 1)}\left(1-(1-u)^{p_{m}}\right)-p_{m}<0 .
$$

When $p=p_{m}$ the time evolves of a step $\Delta_{(m, p)}$ given by $(25)$ :

$$
\Delta_{\left(m, p_{m}\right)}=\ln \left(\frac{a-1}{a-g_{\mathrm{eff}}\left(y_{\left(m, p_{m}\right)}+u x_{\left(m, p_{m}\right)}\right)}\right)=\ln \left(\frac{a-1}{a-g_{\mathrm{eff}} x_{(m, 1)}\left(1-(1-u)^{p_{m}}\right)+p_{m}-1}\right)(
$$

and the resources evolve according to Eq. (26):

$$
x_{(m+1,1)}=1+\left(x_{\left(m, p_{n}\right)}(1-u)-1\right) e^{-\Delta_{\left(m, p_{m}\right)} / \tau_{\mathrm{R}}}=1+\left(x_{(m, 1)}(1-u)^{p_{m}}-1\right) e^{-\Delta_{\left(m, p_{m}\right)} / \tau_{\mathrm{R}}} .
$$

Finally we can plug Eq. (38) into (39) obtaining:

$$
x_{(m+1,1)}=1+\left(x_{(m, 1)}(1-u)^{p_{m}}-1\right)\left(\frac{a-1}{a-g_{\mathrm{eff}} x_{(m+1,1)}\left(1-(1-u)^{p_{m}}\right)+p_{m}-1}\right)^{1 / \tau_{R}}
$$

where $p_{m}$ is the smallest positive integer satisfying Eq. (37). Eq. (40) represents a map for the variable $x_{(m+1,1)}$. The map (40) is shown in Fig. 4 (c.f. captions for details). 

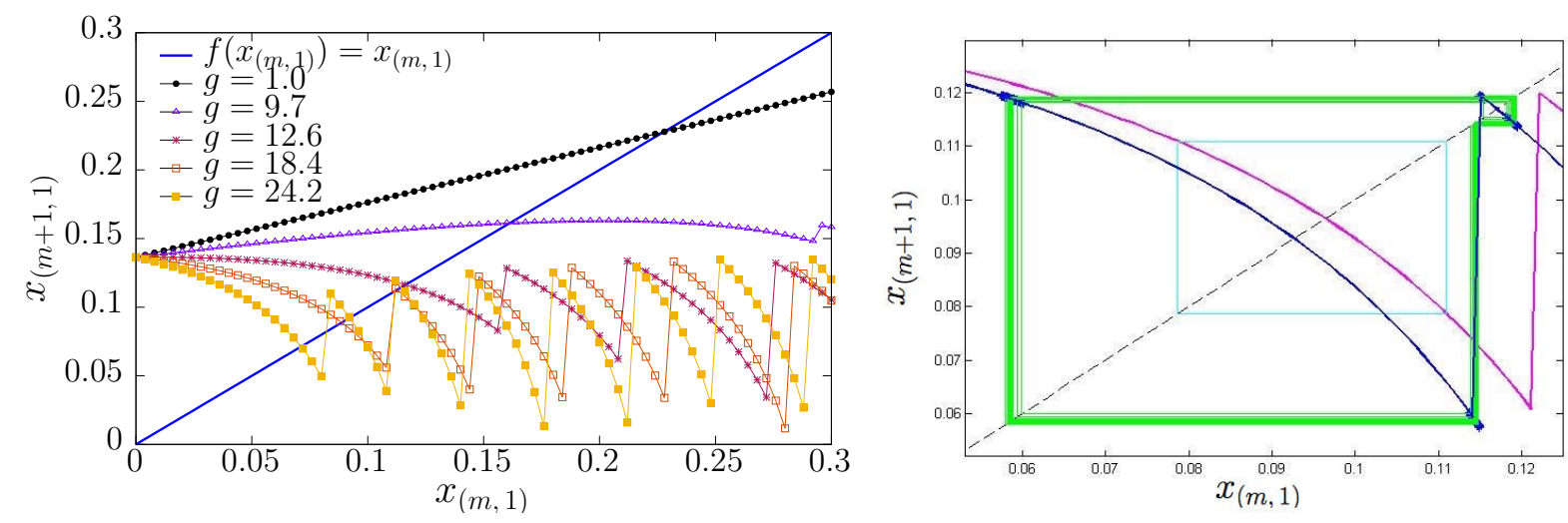

FIG. 4. Left: Map (40) for the MF TUM model with $\tau_{i n}=0$ for different values of $g_{\text {eff }}$ indicated in the legend. Right: in order to highlight the difference between the quasi-synchronous and the bursty transitions, two values of $g_{\text {eff }}$ are plotted in both regimes respectively. For $g_{\text {eff }}=16$ a period-two orbit is observed (light blue), while for $g_{\text {eff }}=30$ - notice that the discontinuity of the map has crossed the $y=x$ line - a chaotic behavior is present (green).

For small values of $g_{\text {eff }}$ the map exhibits a stable fixed point. Increasing $g_{\text {eff }}$, a stable periodtwo orbit is observed (see the second panel of Fig. 4). Finally, at larger values of the coupling, typically when the map features two intersections with the bisector $x_{(m+1,1)}=x_{(m, 1)}$, the dynamical attractor explores a region of the phase space that cannot be confined in a limit cycle, as confirmed by the calculation of the invariant measure that presents a broad distribution (see Fig. 5). This region is strictly chaotic as the Lyapunov exponent of the map is larger then zero, as soon as the limit cycle loses stability. We remark that for $\tau_{\text {in }} \rightarrow 0$ the periodic regime at large values of $g$ does not exist. Indeed, the coupling constant $g$, for $\tau_{\text {in }} \rightarrow 0$, diverges as $g_{\text {eff }} / \tau_{i n}$, while the transition point between the bursty and the asynchronous regimes diverges in the same limit as $s \sim g_{c}^{\text {strong }} \sim \tau_{\text {in }}^{-2}$. 


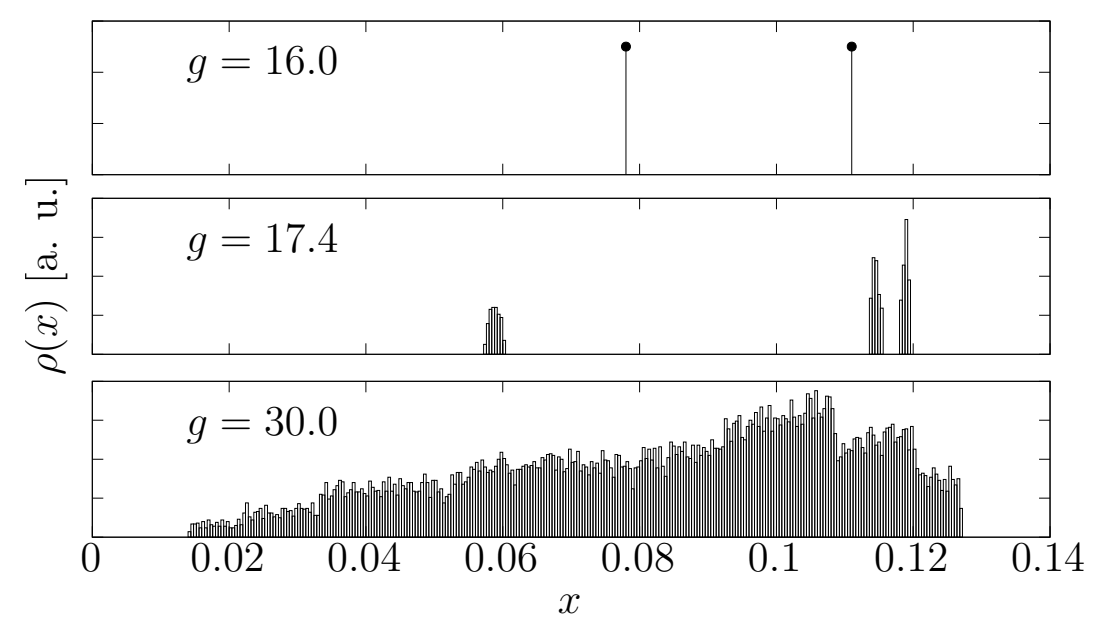

FIG. 5. Probability density $\rho(x)$ of the synaptic resource $x_{n}$, for $g_{\text {eff }}=16$ (upper panel), $g_{\text {eff }}=17.4$ (middle panel) and $g_{\mathrm{eff}}=30$ (lower panel). While two peaks are present for $g_{\mathrm{eff}}=16$, indicating a period-two orbit, a broad distribution, indicating chaotic dynamics, is observed in the last two cases.

\section{DEGREE BASED MEAN FIELD}

In this section we discuss in more detail the numerical analysis of the DMF model. The DMF model consists in $N$ nodes representing neurons with coupling $g k_{i}$ extracted from a probability distribution $P\left(k_{i}\right)$. When not specified differently, the presented numerical results correspond to simulations performed with a Gaussian probability distribution $P\left(k_{i}\right)$, with mean $\mu=0.7$ and variance $\sigma=0.077$.

\section{A. Event driven dynamics simulation protocol}

We have simulated finite size realizations of the event driven dynamics Eq.s (2), with number of neurons $N$. The temporal averages and second moments of the relevant observables (Kuramoto parameter and neuron-averaged interspike interval) are, in principle, a time integral. They have been approximated by a sum evaluated in equispaced times. E.g. $\langle f\rangle=M^{-1} \sum_{m=1}^{M} f\left(t, m \tau+t_{0}\right)$, where $t_{0}$ is a reference initial time and $\tau$ the time spacing. The parameter $\tau$ has been chosen much smaller than the average interspike, and $M$ sufficiently large, so that the results of the averages remain unchanged for larger values of $M$ and for lower values of $\tau$. We have discarded the initial transient regime, whose length $t_{0}$ is strongly dependent on the initial conditions and on the values 
of the parameters. To shorten the length of the transient in series of simulations at different but close values of $g$, we take as an initial condition for the dynamic variables of a simulation at a given $g$, the final configuration attained by the simulation at the immediately lower value $g$. We have also checked the opposite protocol, reducing the value of $g$ and taking as initial condition the final configuration of the precedent simulation, verifying that both protocols lead to identical results. The stationarity of the averages and standard deviations of the Kuramoto parameter and of the interspike interval has been verified comparing the results obtained averaging over temporal windows of exponentially larger and larger width. Moreover, the width $W=\tau M$ of the temporal window is considered to be large enough if the averages of the considered observable over sub-windows of the interval (of size $b \ll W$ ) result statistically uncorrelated (i.e., if not only their average but their fluctuations over different sub-windows do no longer grow significantly with increasing $b$ ). With this method (the jackknife method), we ensure that $W$ is much larger than the correlation time of the considered observable. When we need to distinguish the time average with respect other kind of averages e.g. the average over the different neurons we use the notation $\langle\cdot\rangle_{t}=\langle\cdot\rangle$

\section{B. Global Synaptic field}

Fig. 6 shows the average global synaptic field received by the neurons $g\langle Y\rangle$. It is an increasing function of $g$. As we expect from our arguments in Sec. II A, the transition between quasi-periodic/bursty and bursty/asynchronous regimes occur when $g\langle Y\rangle$ is of the same order of magnitude of the leakage term $a-v(t) \sim O(1)$. In particular, the value of $g\langle Y\rangle$ remains of order $a-v(t)$ (as shown in the first panel in Fig. 6) even for large values of $g$ (e.g. $g \sim 10^{5}$ in the bursty phase), since the resources are typically inactive during the evolution and $\langle Y\rangle$ assumes moderate values (notice that the amount of synaptic current received by the neurons in a given time interval scales as $\left.g \tau_{i n}\right)$. The second panel in Fig. 6 shows that the temporal fluctuations of $g Y(t)$ are larger in the bursty regime. 

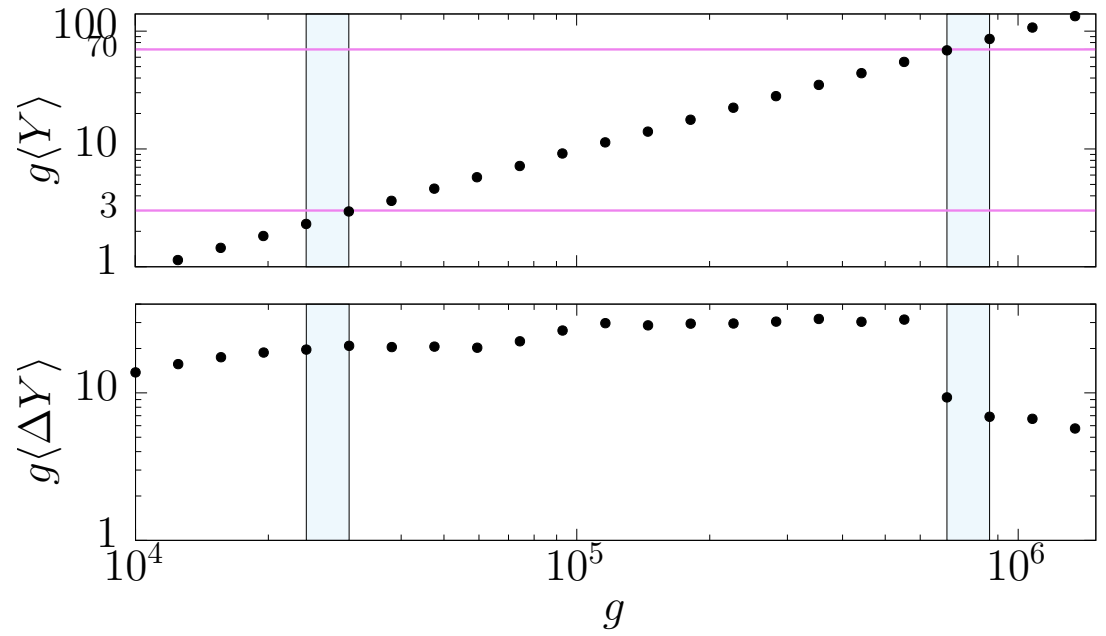

FIG. 6. Higher panel: Average global synaptic field $\langle Y\rangle$ as a function of $g$ in the Gaussian TUM model with $\tau_{i n}=10^{-3}$ and $N=10^{4}$. The vertical stripes indicate the quasi-synchronous/bursty and bursty/asynchronous transitions. The horizontal line indicate the free neuron term $a-v$, revealing the value of $g$ at which the bursty regime takes place. Lower panel: fluctuations of the synaptic field, $\sigma_{Y}$ times $g$.

\section{Fluctuations of the interspike interval, Kuramoto parameter and global field}

Let us call $\Delta_{i}(t)$ the interspike interval corresponding to the last two events of the $i$-th neuron, before the time $t$. The temporal average over the time index $t$, performed as described in Subsec. III A, will be denoted by $\langle\cdot\rangle_{t}$, while the average over the $i$ index will be denoted by $[\cdot]_{i} \equiv(1 / N) \sum_{i=1}^{N}(\cdot)$. In the MF case, the amount of temporal fluctuations of the unique interspike $\Delta_{n}$ around its average is called coefficient of variation, and it is used as an order parameter discriminating among different regimes (see Fig. 2). In the more complicated case of the disordered topology, two kinds of fluctuations of the interspike interval can be defined: those with respect to $t$, averaged over $i$, and those with respect to $i$, averaged over $t$ :

$$
\begin{array}{r}
\sigma_{\Delta}^{2}=\left\langle\left[\Delta_{i}^{2}(t)\right]_{i}-\left[\Delta_{i}(t)\right]_{i}^{2}\right\rangle_{t} \\
{\sigma^{\prime 2}}_{\Delta}^{2}=\left[\left\langle\Delta_{i}^{2}(t)\right\rangle_{t}-\left\langle\Delta_{i}(t)\right\rangle_{t}^{2}\right]_{i}
\end{array}
$$

While the average interspike interval is $I=\left\langle\left[\Delta_{i}(t)\right]_{i}\right\rangle_{t}=\left[\left\langle\Delta_{i}(t)\right\rangle_{t}\right]_{i}$. We observe (see Fig. 7) 
that for both definitions the fluctuations present an apparent discontinuity at $g$ values compatible with the transition values (vertical stripes), at least for sufficiently large values of $N$, for which $\sigma_{\Delta}$ and $\sigma_{\Delta}^{\prime}$ do no longer depend on $N$ within their error-bars. While in the asynchronous and quasi-synchronous regimes $\sigma_{\Delta} / I<\sigma^{\prime}{ }_{\Delta} / I$, the situation is opposite in the bursty regime, where $\sigma_{\Delta} / I>\sigma^{\prime}{ }_{\Delta} / I$.

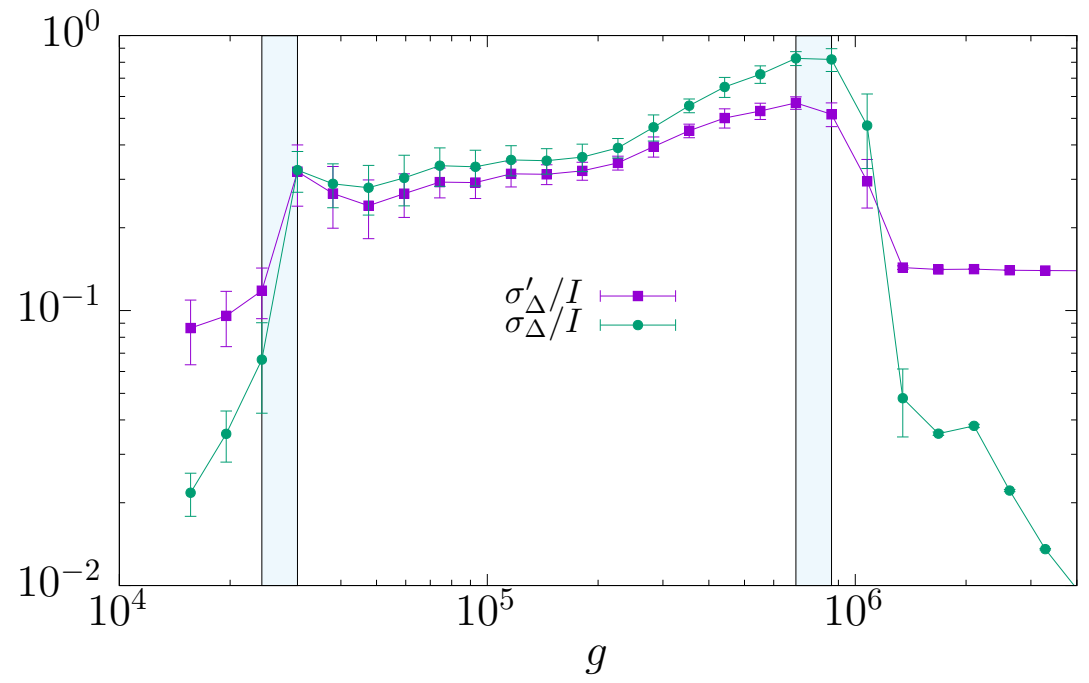

FIG. 7. Two types of fluctuations of the interspike interval $\sigma_{\Delta}$ and $\sigma^{\prime}{ }_{\Delta}$ as a function of $g$, for the DMF model with $N=500, \tau_{i n}=10^{-3}$. The apparent discontinuity of $\sigma_{\Delta}$ and $\sigma^{\prime}{ }_{\Delta}$ does not coincide with the bursty-asynchronous transition: this turns out to be, however, a finite size effect absent for larger values of $N$.

Further insight about the nature of the bursty regime is provided by the Kuramoto parameter $R$ and the global field $Y$ as a function of time. Fig. 8 reveals that these quantities exhibit large temporal fluctuations. 

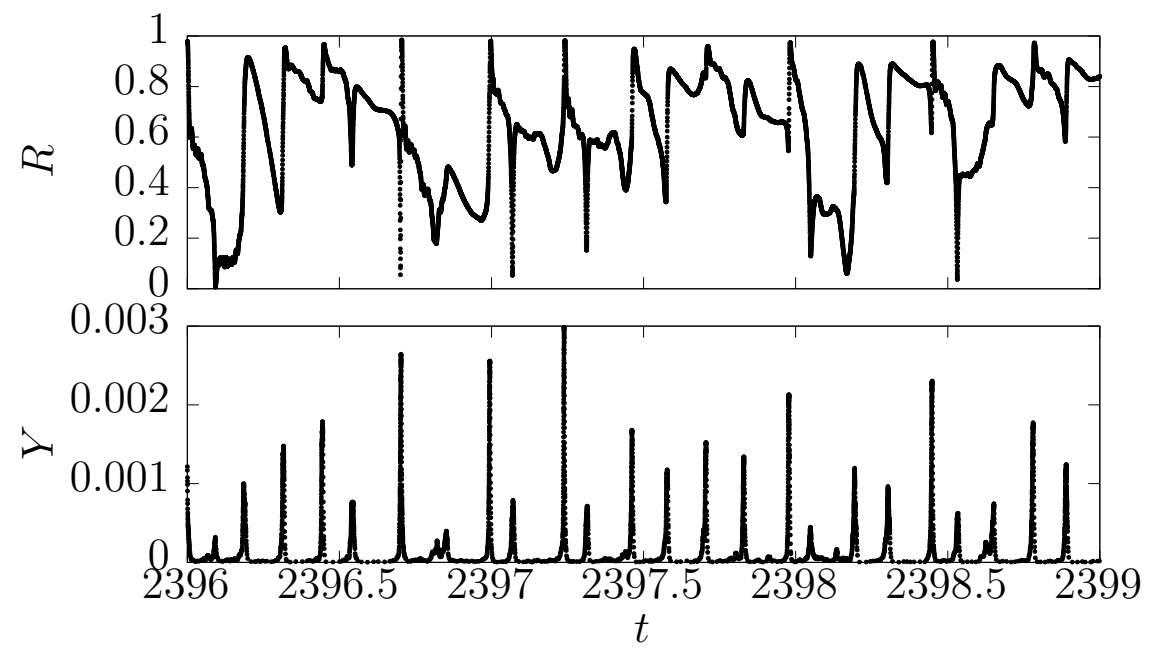

FIG. 8. Kuramoto parameter $R(t)$ (upper panel) and synaptic global field $Y(t)$ (lower panel) as functions of time in the chaotic regime $\left(g=10^{5}, \tau_{i n}=10^{-3}, N=10^{4}\right)$ in the DMF model.

\section{Lyapunov exponents}

The chaotic nature of the bursty dynamical regime is then confirmed by the values of the Lyapunov exponents. Evidence is provided by numerical simulations, showing that the largest Lyapunov exponent remains positive (only) in the bursty phase, even for the largest of the simulated sizes $\left(N=2 \cdot 10^{4}\right.$ for $\left.\tau_{i n}=10^{-3}\right)$, as shown in Fig. 9 for a particular value of $g$. 


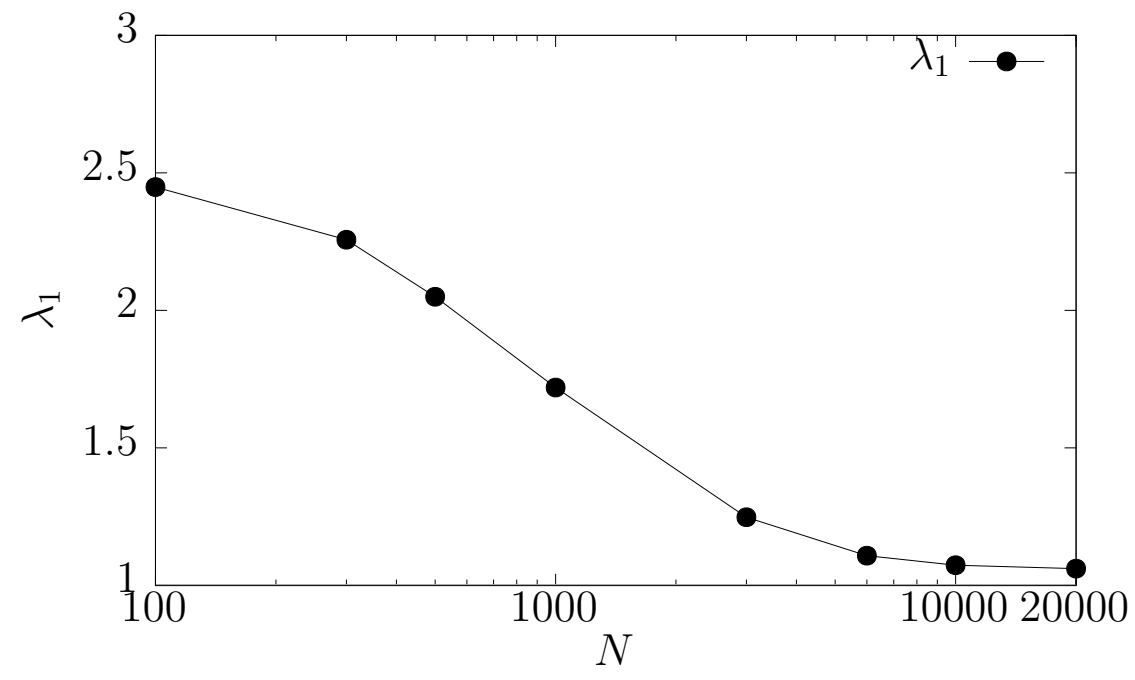

FIG. 9. Finite size scaling of the first Lyapunov exponent $\lambda_{1}$ as a function of the number $N$ of neurons in the DMF approach, with $\tau_{i n}=10^{-3}$ and $g=3 \cdot 10^{4}$.

\section{E. Comparison between the DMF and MF models phase diagrams}

In the DMF model with a non zero variance distribution $P\left(k_{i}\right)$, the two dynamical transitions occurring at $g=g_{1}$ and $g=g_{2}$, between quasi-synchronous/bursty and bursty/asynchronous behaviors, do not coincide exactly with the two corresponding transitions to chaos $g^{\prime}$ and $g^{\prime \prime}$ in the pure MF model. However, in the limit of variance $\sigma \rightarrow 0$, we expect to recover the same transitions of the MF model, as the MF model corresponds to a DMF with a delta function $P\left(k_{i}\right)$. As a numerical confirmation, we have performed a series of simulations of the DMF model with Gaussian $P\left(k_{i}\right)$, with mean $\mu=0.7$ and several decreasing values of $\sigma$. The results, in Fig. 10, show indeed that the transition points $g_{1}$ and $g_{2}$, are compatible for small $\sigma$ with the expected values (horizontal stripes) $g^{\prime}$ and $g^{\prime \prime}$ of the corresponding MF model. The transitions have been estimated by means of the discontinuity of the interspike interval fluctuations. 

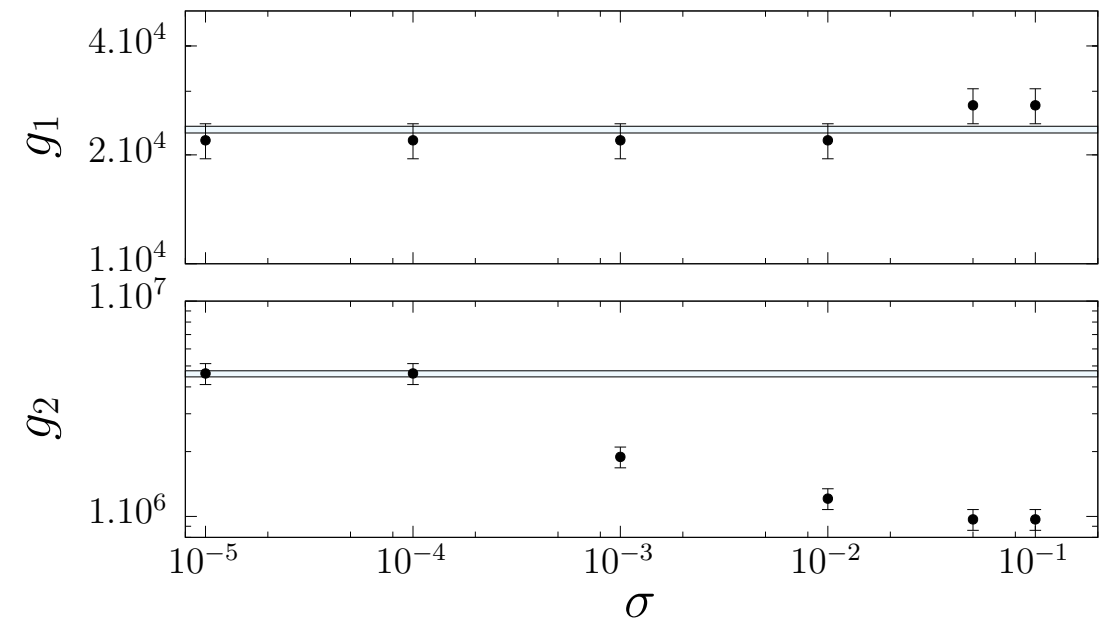

FIG. 10. Transition interval of the synaptic couplings identifying the quasi-synchronous/bursty and bursty/asynchronous transitions (upper and lower panels, $g_{1}$ and $g_{2}$ respectively) for $\tau_{i n}=10^{-3}$, as a function of the variance $\sigma$ of the Gaussian distribution $P\left(k_{i}\right)$, whose average is $\mu=0.7$. The horizontal stripes indicate the two respective values of the transition to chaos, $g^{\prime}$ and $g^{\prime \prime}$, in the MF model.

\section{F. Robustness with respect to the shape of the distribution $P\left(k_{i}\right)$}

In the previous subsection, we verified the robustness of our results with respect to the width $\sigma$ of the Gaussian distribution, by showing that the fully MF behavior is recovered for $\sigma \rightarrow 0$. We remark that a change in the average $\mu$ of the distribution in the DMF Eq.s corresponds to a rescaling of the couplings; in particular the system with average $\mu_{\text {new }}$ displays the same dynamics of the model with average $\mu_{\text {old }}$ but with coupling $g \mu_{\text {new }} / \mu_{\text {old }}$ and variance of the distribution $\sigma \mu_{\text {new }} / \mu_{\text {old }}$. A natural question concerns the robustness of the results with respect to the shape of the coupling distribution. In Fig. 11 we plot the avalanche size distribution and the raster plot for couplings extracted from a Gamma distribution i.e.

$$
P\left(k_{i}\right)=\gamma_{m, \theta}(k)=\frac{1}{\theta^{m} \Gamma(m)} k_{i}^{m-1} e^{-\frac{k_{i}}{\theta}},
$$

where $\Gamma(m)$ is the Euler Gamma function. Fig. 11 shows that the bursty regime is observed also in this case, in the presence of an asymmetric distribution. 


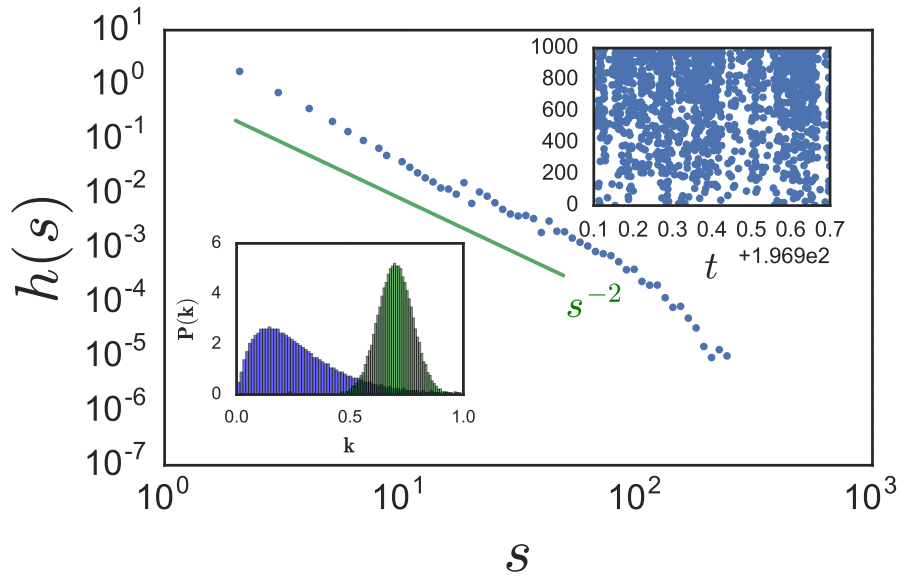

FIG. 11. Numerical results for a Gamma distribution of the couplings $P\left(k_{i}\right)$ defined by Eq. (43) with $m=2$ and $\theta=0.14$. The parameters of the simulations are $a=1.3, g=10^{5}, \tau_{\text {in }}=10^{-3}, \tau_{R}=10$ and the number of neurons is $N=1000$. The main plot represents the distribution $h$ of the avalanche sizes $s$, while the upper inset is the raster plot and the lower inset is a comparison between the Gamma distribution (in blue) and the Gaussian distribution with $\mu=0.7$ and $\sigma=0.077$ (in green).

\section{G. Robustness with respect to the single neuron time scale}

The phase diagram we obtained so far has been described in terms of the coupling constant $g$ and of the synaptic time scales $\tau_{i n}$ and $\tau_{R}$. However, the system is characterized by another natural time scale, i.e. the oscillation period $T=\log (a /(a-1))$ of an isolated neuron. In our simulations $a=1.3$ so that $T \approx 1$. Furthermore, another time scale is present in the system, the membrane time constant $\tau_{m}$ that rescales all time scales of the model. In practice, in order to obtain the physical value of a time scale, we need to multiply such value for $\tau_{m}$, and in physical system $\tau_{m} \approx 20-80 \mathrm{~ms}$.

Letting $a \rightarrow 1$ one obtains a much slower dynamics for the isolated neuron. Physically, a change in $a$ corresponds to a variation of the leakage current, which does not correspond to a trivial redefinition of the time scales since the parameter $a$ drives the non linearity of the evolution, that is the time of the resetting to zero of the potential $v_{i}$. In Fig. 12 we show the raster plot and the avalanches size distribution for a system with $a=1.0001\left(T \approx 9.2\right.$, i.e. if $\tau_{m}=30 \mathrm{~ms}$ we consider a neuron firing intrinsically at $3.5 \mathrm{~Hz}$ ). In this case the bursty regime is recovered for $\tau_{\text {in }}=0.1$, so it is not necessary that $\tau_{i n} \ll 1$. We remark that experimental data analysis about receptors in 
the cortex suggest that the inactivation time is around $3 \mathrm{~ms}$ (i.e. $\tau_{i n}=0.1$ if $\left.\tau_{m}=30 \mathrm{~ms}\right)^{9,10}$.

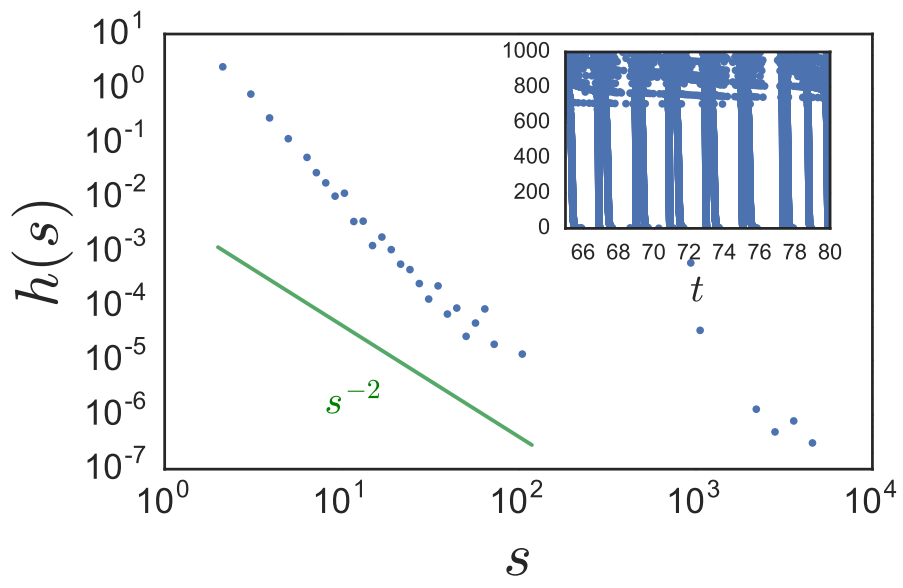

FIG. 12. Numerical results for a system with $a=1.0001, g=120, \tau_{i n}=10^{-1}, \tau_{R}=10, N=1000$. The coupling distribution $P\left(k_{i}\right)$ is a Gaussian with average $\mu=0.7$ and variance $\sigma=0.077$. The main plot represents the distribution $h$ of the avalanches sizes $s$, while the inset is the raster plot.

\section{H. Degree based Mean Field and finite connectivity systems}

The results of this paper have been obtained in the framework of DMF model. In previous works $^{1,11}$ it has been shown that such an approach can be used to study also systems with large but finite connectivities; i.e. neural networks where the interactions are described by a directed adjacency matrix $A_{i, j}$ with a large enough number of incoming synapse $k_{i}^{i n}=\sum_{j} A_{i, j}$. In this case the first of Eq.s (1) reads:

$$
\dot{v}_{i}(t)=a-v_{i}(t)+\frac{g}{N} \sum_{j \neq i}^{N} A_{i, j} y_{j}(t)
$$

while the last two remain unchanged.

The previous papers ${ }^{1,11}$ refer only to the synchronous and the asynchronous regimes. In Fig. 13 we show the raster plot and the avalanches size distribution in a finite connectivity neural network in the bursty regime. In particular, we focus on a random directed network where for each node the number of incoming connections is chosen from a Gaussian distribution $P^{\prime}\left(k_{i}^{i n}\right)$ with mean $N \mu$ and standard deviation $N \sigma$ and we set the parameters to a value where the DMF model exhibits burstyness. Simulations show that also in a finite connectivity networks, where a metric can be introduced, the dynamics is bursty and the avalanche distribution is characterized by a power law 
whose exponent is approximatively -2 .

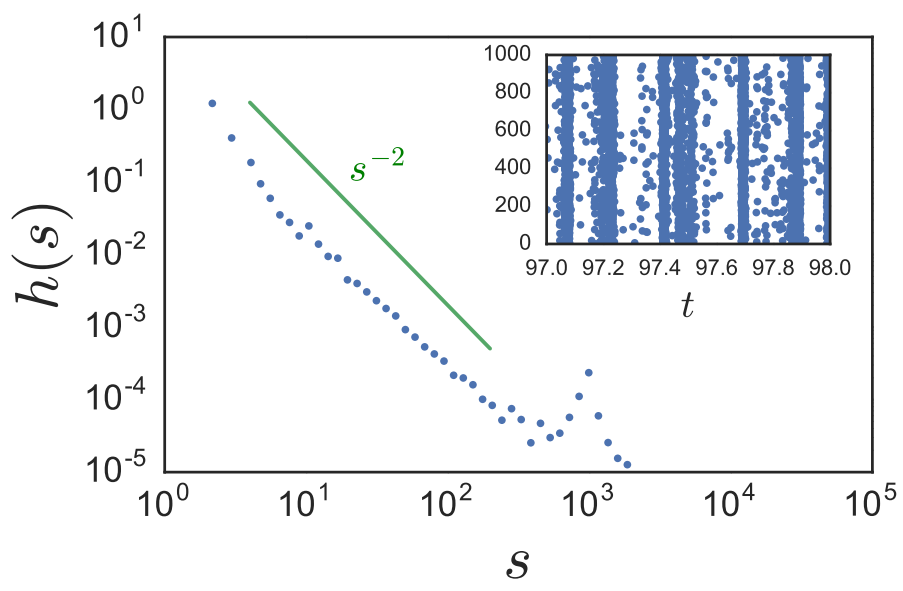

FIG. 13. Numerical results for a random finite connectivity network whit $a=1.3, g=10^{5}, \tau_{\text {in }}=10^{-3}$, $\tau_{R}=10, N=1000$. The incoming degree distribution $P^{\prime}\left(k_{i}^{i n}\right)$ is a Gaussian with average $\mu=0.7 N$ and variance $\sigma=0.077 N$. The main plot represents the distribution $h$ of the avalanches sizes $s$, while the inset is the raster plot.

\section{Temporal correlations}

In the main article, we have defined the connected correlation function of the $N$-neuron system:

$$
\begin{gathered}
C_{N}(\delta)=\left|\left\langle c_{N}(\delta, t)\right\rangle_{t}\right|-\left|\left\langle c_{N}(T, t)\right\rangle_{t}\right| \\
c_{N}(\delta, t)=(1 / N) \sum_{n=1}^{N} e^{\imath \phi_{i}(t)} e^{-\imath \phi_{i}(t+\delta)}
\end{gathered}
$$

where $c_{N}$ is the complex correlation, $\imath$ is the imaginary unit, and $\phi_{i}(t)$ is the Kuramoto phase of neuron $i$ at time $t$. The average over the time variable $\langle\cdot\rangle_{t}$ is performed as described in Subsec. III A.

The connected correlation, $C$, is obtained from the complex correlation by time averaging and by subtracting its asymptotic value $\left|\left\langle c_{N}(\mathcal{T}, t)\right\rangle_{t}\right|$ at a sufficiently high value of $\mathcal{T}$, such that $c_{N}$ does no longer significantly (beyond its fluctuations) depend on $\mathcal{T}$. At this point it is worth mentioning a delicate aspect in the numerical estimation of the asymptotic value of the correlation. In the asynchronous regime, the (disconnected) complex correlation $c_{N}(\delta, t)$ decreases very fast 
for moderate values of $\delta$, of the order of the average interspike interval. The determination of the asymptotic correlation results therefore unambiguous. In the quasi-synchronous regime, we expect $c_{N}$ to achieve a non zero value for arbitrarily large values of $\delta$, at least in the large- $N$ limit, since there is a set $\mathcal{S}$ of synchronous neurons presenting a constant (and independent on time) period. In this way their Kuramoto phase delays $\phi_{i}(t)-\phi_{i}(t+\delta)$ is constant for all $\delta$ and $i \in \mathcal{S}$ and Eq. (45) remains finite for any $\delta^{11}$.

For finite values of $N$, however, the period of synchronized neurons is not exactly constant in time nor in the neuron index (a fact that has been related with the weak chaos exhibited by the model $\left.{ }^{1,11}\right)$. This reflects in the fact that, in the quasi-synchronous regime, $\left|c_{N}(\delta, t)\right|$ rapidly decays, as a function of $\delta$, and begins, as expected, to oscillate around a nonzero value which, however, begins to slowly decrease for larger values of $\delta$ (the slower the larger the value of $N$ ). For large $N$ and moderate $\delta$, this phenomenon is not observed in our numerical calculations, but it becomes an issue in the numerical estimation of the asymptotic value at a very large time delay $\mathcal{T}$. To construct the upper panel of Fig. 5 of the main article, we have estimated the average value of $\left|c_{N}(\mathcal{T}, t)\right|, \mathcal{T}$ being twice the maximum value of $\delta$ used in the figure abscissa, for which the asymptotic value still does not change significantly by doubling $N$ or $M$ (although, we warn, this value is not the stationary value for arbitrarily large values of $\mathcal{T}$ or $M$ ). In other words, to compute the quantity $C_{N}(t)$ in the quasi-synchronous regime, we have assumed that the quantity $c_{N}$ stays constant in $\delta$ for infinite $N$ (an hypothesis with which our numerical data is compatible). A similar (but less significant for the final shape of $C_{N}$ ) strong finite size effect is found in the bursty regime for large values of $\delta$. In any case, the asymptotic value reached by $\left|c_{N}(\delta, t)\right|$ for moderate values of $\delta$, changes abruptly with $g$ : in the synchronous regime it is close, but not equal, to the squared Kuramoto parameter; for $g$ in the bursty regime, it decreases towards a number smaller than the oscillation amplitude.

We end this section proposing an alternative definition of temporal correlation function: $C_{N}^{\prime}(\delta)=\left|\left\langle K_{N}(t) K_{N}(t+\delta)^{*}\right\rangle_{t}\right|$, where $K_{N}=(1 / N) \sum_{i=1}^{N} \exp \left(\imath \phi_{i}(t)\right)$ is the (complex) Kuramoto parameter. The qualitative behavior of both definitions is quite similar (they differ in the fact that the first definition, Eq. (44) accounts for the correlations between neurons with equal coupling $k_{i}$ only), although the finite size behavior of the latter definition results to be slower in our numerical analysis; we have consequently used the first definition to draw our conclusions about the temporal correlations of the system in the main article. 


\section{J. Kolmogorov Complexity}

As mentioned in the main article, we have considered the Kolmogorov complexity (KC) of the raster plot of the Gaussian model, for several values of $g$ across three model regimes. In particular, we consider the sequence of differences of spike times $\Delta_{n}$ (not writing the spiking neuron index), and estimate ${ }^{12}$ the $\mathrm{KC}$ of such sequences as the size of the zipped file containing the sequence, divided by the size of the original file. In practical terms, we use the gzip algorithm to compress the file containing a sequence of $L$ spiking times (written in ascii with constant number of digits), corresponding to a simulation of the TUM model with a given set of parameters $\{\sigma\}$. We then

compute $K_{L}$ as the ratio of the compressed file size over the original file size. We avoid writing in the original file data corresponding to the transient, by skipping a large enough amount of initial events. In this circumstance, we observe that, for large enough number of events (i.e., of lines in the original file) $L, K_{L} \simeq K$ does not depend significantly on $L$, we take then $K$ as the estimation of the $\mathrm{KC}$ of $\{\sigma\}$. The number of digits $n$ used to store the spiking times being large enough, has the mere influence of shifting the whole $K$ versus $\{\sigma\}$ curve by an $n$-depending constant.

In the presence of a quasi-synchronous to asynchronous regime transition, the $\mathrm{KC}$ presents a maximum at the parameters $\{\sigma\}$ corresponding to the transition value. This arises for a variety of types of integrate-and-fire models, as we will show in a forthcoming publication. In the presence of the bursty regime presented and characterized in this work, one observes that the bursty regime presents a higher value of the $\mathrm{KC}$, the maximum of which is found for values of $g$ in the bulk bursty regime, i.e. larger than the quasi-synchronous/asynchronous transition value.

\section{K. Avalanche size distribution}

In the main article we have presented the avalanche size distribution $h(s)$ of the DMF, exhibiting power law behavior in the bursty regime. To compute $h(s)$, the avalanche is defined as a set of consecutive neuronal spikes such that all the interspike intervals of such set, $\left\{\Delta_{n}\right\}_{n}$, satisfy $\Delta_{n}<\delta_{\mathrm{t}}$, where $\delta_{\mathrm{t}}$ is a threshold. The avalanche size distribution is consequently taken among various avalanche events during the temporal evolution. We have numerically checked that, for the cases of interest, there is a wide range of the threshold $\delta_{\mathrm{t}}$ (of at least two orders of magnitude), for which the avalanche size distribution does not depend significantly on it (see the left panel of Fig. 14).

With this definition, the distribution of avalanche sizes, $h(s)$, becomes broadly distributed, 
as shown in Fig. 3 of the main article, and it is compatible with a power law behavior for a wide range of values of $g$ in the bursty phase $\left(\left[5.10^{4}: 10^{6}\right]\right.$, for $\tau_{i n}=10^{-3}$ and $\left.N=10^{4}, 6.10^{3}\right)$, in a range of the size $s$ of roughly three orders of magnitude (see Fig. 14, right panel). The exponent $\gamma$, obtained by means of a linear fit in logarithmic scale, results to assume the values $\gamma_{1}=-2.39 \pm 0.07, \gamma_{2}=-2.07 \pm 0.04$ and $\gamma_{3}=-2.08 \pm 0.05, \gamma_{4}=-2.1 \pm 0.3$ for $g_{1} \simeq 1.160 \cdot 10^{5}$, $g_{2} \simeq 2.263 \cdot 10^{5}, g_{3} \simeq 4.417 \cdot 10^{5}, g_{4}=1.07 \cdot 10^{6}$, respectively, for $N=10^{4}$, and: $\gamma_{1}=-2.42 \pm 0.25$, $\gamma_{2}=-2.07 \pm 0.06$ and $\gamma_{3}=-2.04 \pm 0.05$ for $N=3 \cdot 10^{3}$ and the same values of $g$. The error intervals are calculated as the interval of $\gamma$ values for which the fit (performed in the intercept only) results to exhibit a value of the sum of squared residuals per degree of freedom lower than one. The fit takes into account the errors of the histogram points $h\left(s_{i}\right)$ (shown as error bars in the figure), which in their turn are calculated with a jackknife error estimation procedure: one blocks the data in blocks of sufficiently large size $b$, and estimates the error of $h\left(s_{i}\right)$ as three times the standard deviation among the different histogram points in different blocks, $\left\{h_{j}\left(s_{i}\right)\right\}_{j}$.
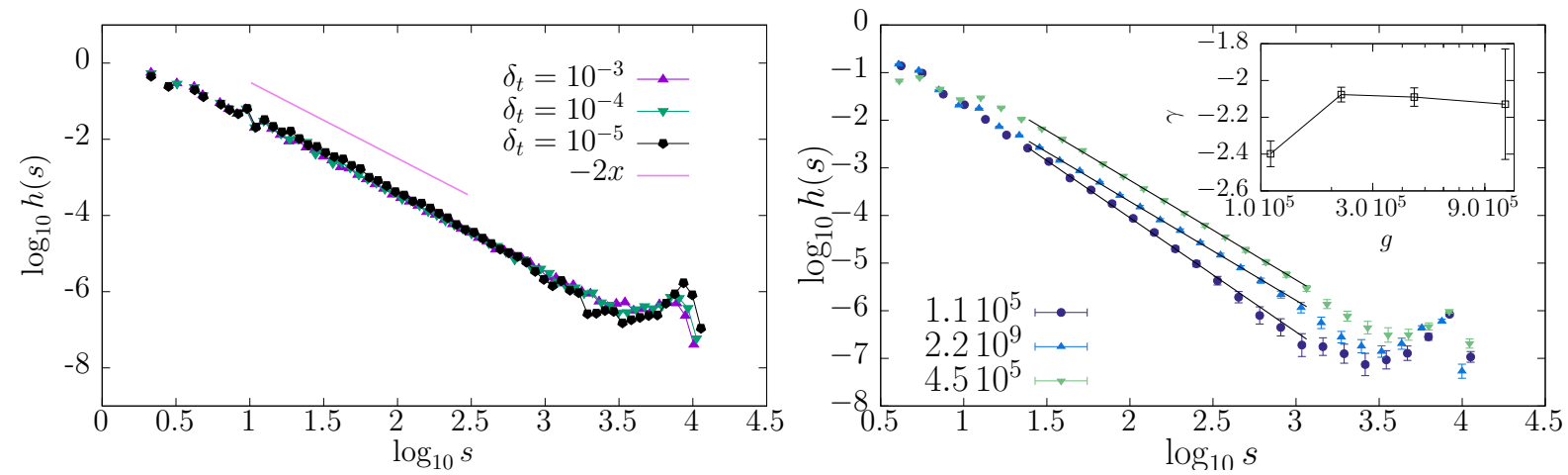

FIG. 14. Left panel: avalanche size distribution of the DMF model with $\tau_{i n}=10^{-5}, N=10^{4}$, $g=10^{7}$, for three values of the threshold $\delta_{t}$. Right panel: Avalanche size distribution for the system with $\tau_{i n}=10^{-3}, N=10^{4}$ versus $g$. The straight lines are linear fits in an interval indicated by the x-axis range of the lines (the interval used for $g_{4} \simeq 1.07 \cdot 10^{6}$ being $[2: 3.5]$ ). The inset shows the value $\gamma$ of the resulting slope versus $g$, for $N=10^{4}$.

* alessandro.vezzani@fis.unipr.it

1 R. Burioni, M. Casartelli, M. di Volo, R. Livi, and A. Vezzani, Sci. Rep. 4, 4336 (2014).

2 R. Brette, Neural Comput. 19, 2604 (2007). 
3 M. di Volo, R. Livi, S. Luccioli, A. Politi, and A. Torcini, Phys. Rev. E 87, 032801 (2013).

4 M. Ding, and W. Yang, Phys.Rev. E 56, 4009 (1997).

5 J.F. Heagy, T.L. Carol, and L.M. Pecora, Phys.Rev. E 50, 1874 (1994).

6 G. Benettin, L. Galgani, A. Giorgilli, and J. M. Strelcyn, Meccanica 15, 9 (1980).

7 I. Shimada, and T. Nagashima, Progr. Theor. Phys. 61, 1605 (1979).

8 A. Levina, J. M. Herrmann, and T. Geisel, Nat. Phys. 3, 857 (2007).

9 M. Tsodyks, and H. Markram, PNAS 94, 719-23 (1997).

10 M. Tsodyks, K. Pawelzick, and H. Markram, Neural Comput. 15, 821-35 (1998).

11 M. di Volo, R. Burioni, M. Casartelli, R. Livi, and A. Vezzani, Phys. Rev. E 90, 022811 (2014) .

12 A. Kaitchenko, in Canadian Conference on Electrical and Computer Engineering, (IEEE, 4, pg. 2255 2004). 